\title{
A NEW TWO-DIMENSIONAL SHALLOW WATER MODEL INCLUDING PRESSURE EFFECTS AND SLOW VARYING BOTTOM TOPOGRAPHY
}

\author{
Stefania Ferrari ${ }^{1}$ And FAusto SALERI ${ }^{2}$
}

\begin{abstract}
The motion of an incompressible fluid confined to a shallow basin with a slightly varying bottom topography is considered. Coriolis force, surface wind and pressure stresses, together with bottom and lateral friction stresses are taken into account. We introduce appropriate scalings into a three-dimensional anisotropic eddy viscosity model; after averaging on the vertical direction and considering some asymptotic assumptions, we obtain a two-dimensional model, which approximates the three-dimensional model at the second order with respect to the ratio between the vertical scale and the longitudinal scale. The derived model is shown to be symmetrizable through a suitable change of variables. Finally, we propose some numerical tests with the aim to validate the proposed model.
\end{abstract}

Mathematics Subject Classification. 35L65, 65M60.

Received: January 21, 2003.

\section{INTRODUCTION}

Simulation of free surface flows plays an important role in many engineering applications such as in the coastal exploitation and navigation. Classical mechanics (see e.g. [12]) describes the flow of a Newtonian, viscous and incompressible fluid through the three-dimensional Navier-Stokes equations (here called "NS3d" equations). It is well known that the numerical solution of NS3d equations for a free surface flow is generally very onerous. For this reason, in order to make the simulation of the water level as efficient as possible, when the ratio between the vertical and the horizontal scales is small, it is usual to consider the so called "Shallow Water" approximation.

In the literature (see e.g. $[1-4,14]$ ), in order to obtain a 2D Shallow Water approximation of NS3d model, atmospheric pressure and wind effects on the free surface, bottom topography and friction effects on the bottom through the Chézy law are taken into account; the viscosity is neglected in the derivation and a posteriori is added in the Shallow Water model by the so-called efficient-viscosity. The derivations of such models are heuristic and often unclear, even though they have been used in hydraulics for over twenty years. In particular, we will refer as classical Shallow Water model to the 2D model presented in [1].

\footnotetext{
Keywords and phrases. Navier-Stokes equations, Saint Venant equations, free surface flows.

1 MOX, Dipartimento di Matematica F. Brioschi, Politecnico di Milano, via Bonardi 9, 20133 Milano, Italy.

e-mail: Stefania.Ferrari@polimi.it

2 MOX, Dipartimento di Matematica F. Brioschi, Politecnico di Milano, via Bonardi 9, 20133 Milano Italy.

e-mail: Fausto.Saleri@polimi.it
} 
In [7] Gerbeau and Perthame started from the free surface two-dimensional Newtonian Navier-Stokes equations, taking friction effects on the (flat) bottom through a Navier condition into account and gravity force as the only volume force. No stresses on the free-surface were taken into account and no turbulence model for the stress tensor was considered, although the Reynolds number associated to the physical phenomenon is very large. The effect of the viscosity is recovered in the modelling of the friction term of zeroth order and replaces the classical Chézy term. They derived rigorously a 1D Shallow Water model which approximates the free surface two-dimensional newtonian Navier-Stokes equations at the second order with respect to the ratio between the vertical and the longitudinal scales.

A lot of articles are also present in the literature concerning the numerical discretization of systems of Shallow Water type, see e.g. Jin in [15] and Kurganov et al. in [16] .

In the present paper we improve the classical 2D Shallow Water model by deriving a new 2D Shallow Water model, extending the rigorous derivation presented in [7]; we thus obtain a $2 \mathrm{D}$ hyperbolic system. We won't face to the difficulties of discretizing an hyperbolic system as in [15] or in [16]; in fact we will perturb our system through an "artificial" viscous term of second order and then deal (about numerical discretization) with a parabolic system as in [6].

We will start from the NS3d equations including a non-flat bottom topography with small slopes; a friction force is taken into account on the bottom and modeled through a Navier condition. Surface stresses are considered on the free surface, given both by atmospheric pressure and wind. It is important to notice that considering atmospheric pressure effects allows the simulation of real phenomena such as storms, where the varying of that quantity is compulsory. In our work it is worth noting the originality of treating atmospheric pressure, where we "plugged" it into a free surface (reduced) model, while in the oceanographic literature (see e.g. [8]), rigid lid models are considered. Gravity force and Coriolis force both act on the fluid as volume forces. We also add a turbulence model for the stress tensor in NS3d, which we consider to present anisotropy with respect to the vertical and the longitudinal scale: therefore two different eddy viscosities (vertical and longitudinal) are introduced following [18]. The effect of the eddy (vertical) viscosity is recovered in the modelling of the friction term of zeroth order and replaces the classical Chézy term. The approximation between the physical NS3d model and our new model as well as the approximation between the physical stress tensor arising from NS3d equations and the approximated stress tensor arising from our model, is of the second order with respect to the ratio between the vertical and the longitudinal scales. We will refer to our new Shallow Water 2D model as the SW2d model.

This paper is organized as follows. In Section 2 we provide the derivation of the conservative model SW2d. In Section 3 the symmetrization of SW2d model, the SSW2d model in a not-conservative form is presented; we also discuss there about second order viscous perturbations of SSW2d model and about the convergence of its solution to the one of the unperturbed problem, as the perturbation vanishes. In Section 4 some preliminary numerical experiments on test cases are carried out in order to compare SSW2d model with respect to the classical Shallow Water model.

\section{Derivation of the SW2D MOdel}

Let us consider an incompressible fluid with constant density $\rho>0$ in a three dimensional domain $\mathcal{U}$ which is normal with respect to the vertical direction $z$ and vertically bounded by the surfaces $z=\eta(\mathbf{x}, t)$ and $z=b(\mathbf{x})$ :

$$
\mathcal{U}:=\{(t, x, y, z): t \in(0, T),(x, y) \in \Omega, z \in(b(x, y), \eta(t, x, y))\},
$$

where $T>0, \eta:[0, T] \times \bar{\Omega} \rightarrow \mathbb{R}(T>0)$ denotes the elevation, $b: \bar{\Omega} \rightarrow \mathbb{R}^{-}$the bottom depth with respect to the same reference level and $H=\eta-b$ the total height of the fluid from the bottom to the free surface 


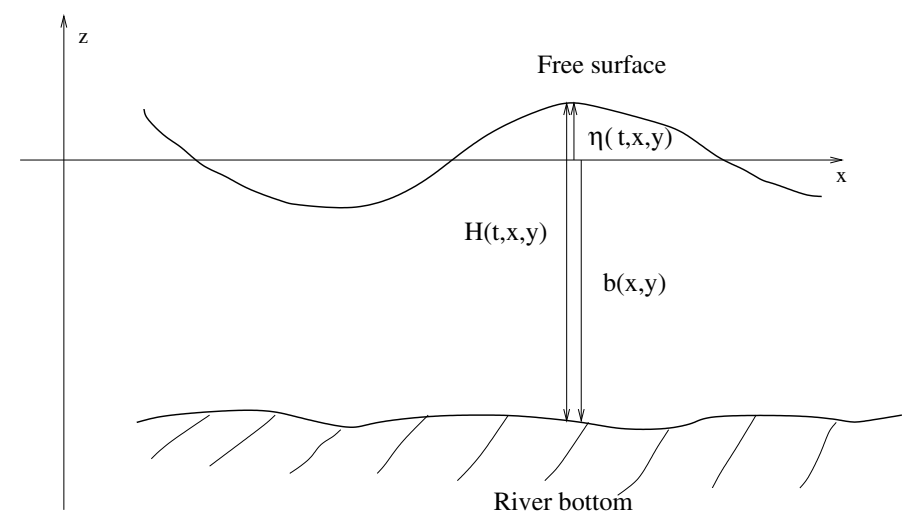

FiguRE 1. Vertical cross-section of the domain.

(see Fig. 1). We denote by $\Omega$ the projection of $\mathcal{U}$ on the $x y$-plane and, for the sake of simplicity, we assume $\Omega$ be the following rectangular domain:

$$
\Omega=\left\{(x, y) \in \mathbb{R}^{2}: y \in\left(-L_{2} / 2, L_{2} / 2\right), x \in\left(x_{\text {in }}, x_{\text {out }}\right)\right\},
$$

where $L_{2}>0$ and $x_{\text {in }}<x_{\text {out }}$ are the inflow and outflow abscissae of the portion of fluid flow, we are focusing on. In fact we consider our fluid flow to cover a total lenght $L_{1}$ much larger than the segment $\left(x_{\text {in }}, x_{\text {out }}\right)$.

The governing equations for the motion of an incompressible fluid in $\mathcal{U} \times(0, T], T>0$, are the Navier-Stokes equations (in the sequel, NS3D) that can be written as:

$$
\left\{\begin{array}{l}
\frac{\partial u}{\partial t}+\frac{\partial u^{2}}{\partial x}+\frac{\partial(u v)}{\partial y}+\frac{\partial(u w)}{\partial z}+\frac{1}{\varrho} \frac{\partial p}{\partial x}=l v+\frac{1}{\varrho}\left(\frac{\partial \sigma_{11}}{\partial x}+\frac{\partial \sigma_{12}}{\partial y}+\frac{\partial \sigma_{13}}{\partial z}\right) \\
\frac{\partial v}{\partial t}+\frac{\partial(u v)}{\partial x}+\frac{\partial v^{2}}{\partial y}+\frac{\partial(v w)}{\partial z}+\frac{1}{\varrho} \frac{\partial p}{\partial y}=-l u+\frac{1}{\varrho}\left(\frac{\partial \sigma_{21}}{\partial x}+\frac{\partial \sigma_{22}}{\partial y}+\frac{\partial \sigma_{23}}{\partial z}\right) \\
\frac{\partial w}{\partial t}+\frac{\partial(u w)}{\partial x}+\frac{\partial(v w)}{\partial y}+\frac{\partial w^{2}}{\partial z}+\frac{1}{\varrho} \frac{\partial p}{\partial z}=-g+\frac{1}{\varrho}\left(\frac{\partial \sigma_{31}}{\partial x}+\frac{\partial \sigma_{32}}{\partial y}+\frac{\partial \sigma_{33}}{\partial z}\right) \\
\nabla \cdot \boldsymbol{v}=0
\end{array}\right.
$$

where $\boldsymbol{v}=(u, v, w)^{T}$ and $p$ are the velocity and the pressure fields, respectively, $l$ is the Coriolis coefficient and $g$ is the gravity acceleration (oriented downward). To obtain an appropriate form for the stress tensor $\boldsymbol{\sigma}$ appearing in (2.3), we introduce a turbulence model which is given trough a linear constitutive relationship between $\boldsymbol{\sigma}$ and the strain-rate tensor $\mathbb{D}$, which is the traceless, symmetric tensor given by:

$$
\mathbb{D}=\nabla \boldsymbol{v}+(\nabla \boldsymbol{v})^{T}
$$

Following Levermore and Sammartino in [18], we observe that this relationship cannot be assumed to be isotropic because the horizontal and the vertical length scales will be of different orders in a shallow water approximation. Because the scale of the horizontal eddies will be much larger than that associated with vertical eddies, the eddy viscosity associated with horizontal eddies $\left(\mu_{h}\right)$ will be much larger than that associated with vertical eddies $\left(\mu_{v}\right)$, i.e. $\mu_{h}>>\mu_{v}$ (see also [19]). The relationship should be symmetric with respect to the vertical direction; that is to say, it should be invariant under rotations about a vertical axis. In [18] authors introduced a further eddy viscosity, say the bulk viscosity $\mu_{e}$ and they made the physical assumption that $\mu_{e}$ should be much smaller than $\mu_{h}$; but they were dealing with the rigid lid approximation; in our case, without any rigid lid approximation, we take $\mu_{h}$ and $\mu_{e}$ of the same order of magnitude. 
The null-trace symmetric stress tensor $\boldsymbol{\sigma}$ is therefore given by:

$$
\boldsymbol{\sigma}=\left(\sigma_{i j}\right)=\left[\begin{array}{lll}
\mu_{h} \mathbb{D}_{11} & \mu_{h} \mathbb{D}_{12} & \mu_{v} \mathbb{D}_{13} \\
\mu_{h} \mathbb{D}_{21} & \mu_{h} \mathbb{D}_{22} & \mu_{v} \mathbb{D}_{23} \\
\mu_{v} \mathbb{D}_{31} & \mu_{v} \mathbb{D}_{32} & \mu_{h} \mathbb{D}_{33}
\end{array}\right] .
$$

As usual, the physical total stress tensor $\boldsymbol{\sigma}_{\boldsymbol{T}}$ (symmetric), is obtained adding pressure to $\boldsymbol{\sigma}$ as:

$$
\boldsymbol{\sigma}_{T}=-p \boldsymbol{I}+\boldsymbol{\sigma} .
$$

System (2.3) must be completed by the initial condition for the velocity field, for the elevation and by suitable boundary conditions. In particular, the boundary of $\mathcal{U}$ can be split into 5 different sides: the free surface $\mathcal{S}$, the bottom surface $\mathcal{B}$, the inflow surface $\mathcal{L}_{\text {in }}$, the outflow surface $\mathcal{L}_{\text {out }}$ and the closed surface $\mathcal{L}_{c_{ \pm}}$(see Fig. 2).

On $\mathcal{S}$ and on $\mathcal{B}$ both dynamical (involving the stress tensor) and kinematical conditions are set, while on $\mathcal{L}_{\text {in }}$, $\mathcal{L}_{\text {out }}$ and $\mathcal{L}_{c_{ \pm}}$only kinematical conditions will be set. In particular, wind and atmospheric stresses are given on the free-surface. Therefore,

$$
s_{\sup \mid x y}=C|\boldsymbol{W}| \boldsymbol{W}, \quad s_{\sup \mid z}=p_{a},
$$

where $\boldsymbol{s}_{\text {sup }}$ is the total three-dimensional stress acting on $\mathcal{S}, \boldsymbol{W}$ is the wind velocity, $C$ is a suitable coefficient and $p_{a}$ is the (positive) atmospheric pressure.

At the bottom $\mathcal{B}$ we assume that $s_{\mathrm{bfr}}=k \boldsymbol{v}(t, x, y, b)$, where $k>0$ is the friction coefficient. Finally, we assume that the boundary $\boldsymbol{\Gamma}$ of the two-dimensional domain $\Omega(2.2)$ may be partitioned as follows:

$$
\boldsymbol{\Gamma}=\partial \Omega=\overline{\boldsymbol{\Gamma}_{\text {in }} \cup \boldsymbol{\Gamma}_{\text {out }} \cup \boldsymbol{\Gamma}_{\boldsymbol{c}}},
$$

where $\boldsymbol{\Gamma}_{c}=\boldsymbol{\Gamma}_{c+} \cup \boldsymbol{\Gamma}_{c-}$,

$$
\begin{aligned}
& \boldsymbol{\Gamma}_{\text {in }}=\left\{(x, y) \in \boldsymbol{\Gamma}: x=x_{\text {in }}, y \in(-L / 2, L / 2)\right\}, \\
& \boldsymbol{\Gamma}_{\text {out }}=\left\{(x, y) \in \boldsymbol{\Gamma}: x=x_{\text {out }}, y \in(-L / 2, L / 2)\right\}, \\
& \boldsymbol{\Gamma}_{\boldsymbol{c} \pm}=\left\{(x, y) \in \mathbb{R}^{2}: x \in\left(x_{\text {in }}, x_{\text {out }}\right), y=\mp L / 2\right\} .
\end{aligned}
$$

The suffixes in, out and $\boldsymbol{c}$ stay respectively for inflow, outflow and closed, and refer to a possible physical characterization of the boundary edges. Correspondingly, we define:

$$
\mathcal{L}=\left\{(t, x, y, z) \in[0, T] \times \mathbb{R}^{3}:(t, x, y) \in(0, T) \times \boldsymbol{\Gamma}, \quad z \in(b(x, y), \eta(t, x, y))\right\}
$$

and analogously $\mathcal{L}_{\text {in }}, \mathcal{L}_{\text {out }}$ and $\mathcal{L}_{c_{ \pm}}$. Normals to $\mathcal{L}$ are depicted in Figure 2. Following the previous notations, for every $t \in(0, T]$, we complete the Navier-Stokes system with the following set of boundary conditions:

$$
\begin{aligned}
& \text { on } \mathcal{S}:\left\{\begin{array}{c}
\frac{1}{\varrho} \boldsymbol{\sigma}_{\boldsymbol{T}} \cdot \boldsymbol{n}_{\boldsymbol{s}}+\boldsymbol{s}_{\mathrm{sup}}=\mathbf{0}, \\
w=\frac{\partial \eta}{\partial t}+\boldsymbol{v} \cdot \nabla \eta ;
\end{array}\right. \\
& \text { on } \mathcal{B}:\left\{\begin{array}{c}
\frac{1}{\varrho} \boldsymbol{\sigma}_{\boldsymbol{T}} \cdot \boldsymbol{n}_{\boldsymbol{b}}+\boldsymbol{s}_{\mathrm{bfr}}=\mathbf{0}, \\
w=\boldsymbol{v} \cdot \nabla b ;
\end{array}\right. \\
& \text { on } \mathcal{L}: \quad \begin{cases}\boldsymbol{v} \cdot \boldsymbol{n}_{ \pm}=0 & \text { on } \mathcal{L}_{c_{ \pm}}, \\
\boldsymbol{v} \cdot \boldsymbol{n}_{\text {in }}>0 & \text { on } \mathcal{L}_{\text {in }}, \\
\boldsymbol{v} \cdot \boldsymbol{n}_{\text {out }}<0 & \text { on } \mathcal{L}_{\text {out }},\end{cases}
\end{aligned}
$$

where $\mathbf{n}_{\mathbf{k}}$ with $\mathbf{k}$ equal to $\mathbf{b}, \mathbf{s}, \pm$, in and out denotes the outward normals to the bottom, the free-surface and the closed boundaries. 


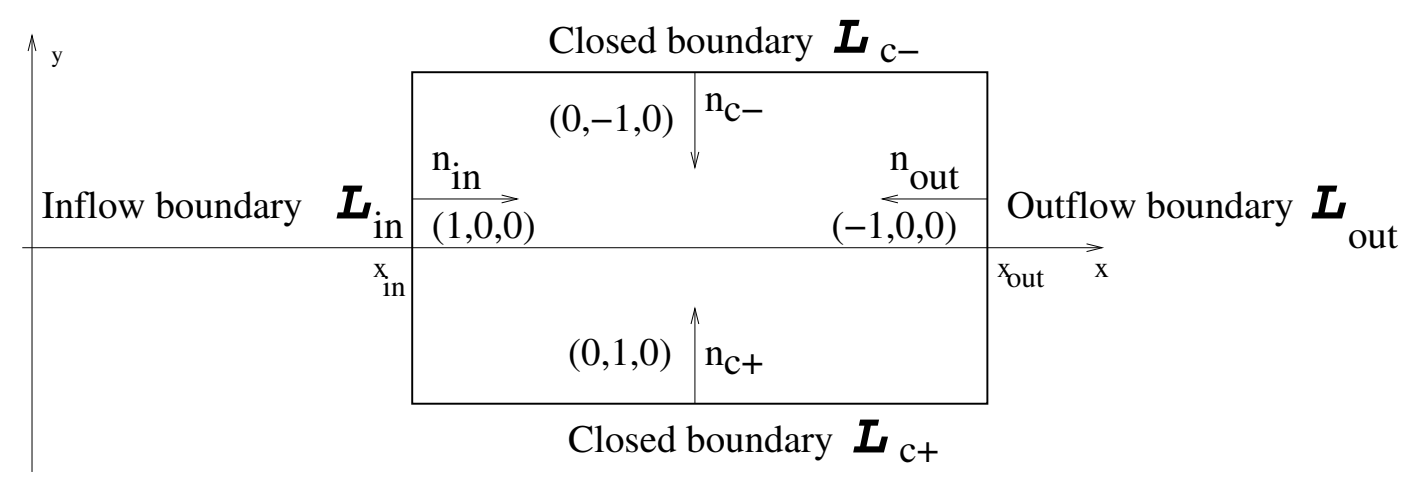

Figure 2. Cut of domain $\mathcal{U}$ at level $z=\bar{z} \in(b(x, y), \eta(t, x, y))$.

\subsection{Adimensionalization of the NS3d system}

In this section, we introduce an adimensional form of the NS3d equations, assuming that the ratio $\varepsilon$ between the vertical and the longitudinal scale is small. In particular, let us consider the following absolute scales:

for the total length: $L_{1}$, for the width: $L_{2}$,

for the height: $A, \quad$ for the $x$-component of the velocity: $U$.

As we are in the Shallow Water assumptions, we consider $A \ll L_{1}$ and $A \ll L_{2}$.

On these assumptions, we set:

$$
\varepsilon:=A / L_{1}, \quad \gamma:=L_{2} / L_{1}, \quad \nu_{h}:=\frac{\mu_{h}}{\rho U L_{1}}, \quad \nu_{v}:=\frac{\mu_{v}}{\rho U L_{1}}
$$

Note that $\nu_{h}$ and $\nu_{v}$ represent the inverse Reynolds numbers with respect to the eddy viscosities $\mu_{h}$ and $\mu_{v}$, while $\gamma$ is considered to be a finite ratio such that $\varepsilon \ll \gamma$. The corresponding derived scales are then:

$$
\begin{aligned}
& \text { for the time: } L_{1} / U, \quad \text { for the } y \text {-component of the velocity: } V=\gamma U, \\
& \text { for the pressure/density: } U^{2}, \text { for the } z \text {-component of the velocity: } W=\varepsilon U \text {. }
\end{aligned}
$$

For the sake of simplicity we indicate again by $\eta, b, H, u, v, w, p / \varrho$, respectively, elevation, bottom level, total distance from the bottom level to the elevation level, velocity components and specific pressure, after rescaling. The gravity acceleration $g$, the Coriolis coefficient $l$, the surface stress $\boldsymbol{s}_{\text {sup }}$ and the friction coefficient $k$, are respectively rescaled as $G, \lambda, \boldsymbol{S}_{\text {sup }}$ and $\alpha$. Moreover, let us denote again by $p_{a}$ the rescaling of the atmospheric pressure.

It is worth mentioning the rescaling criteria for these last quantities. Denoting with square brackets the term whose dimension we need to the rescaling, we have:

$$
\begin{aligned}
& g \longrightarrow G\left[\left[\frac{1}{\varrho} \frac{\partial p}{\partial z}\right]\right]=G \frac{U^{2}}{A}, \quad l \longrightarrow \lambda \frac{U}{L_{2}},
\end{aligned}
$$

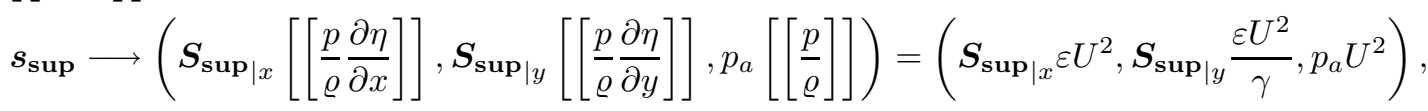

$$
\begin{aligned}
& k \longrightarrow \alpha U
\end{aligned}
$$




$$
\begin{aligned}
\boldsymbol{s}_{\mathrm{bfr}} & =k \boldsymbol{v} \longrightarrow\left(\alpha u\left[\left[\frac{p}{\varrho}\right]\right], \alpha v \gamma\left[\left[\frac{p}{\varrho}\right]\right], \alpha w \varepsilon\left[\left[\frac{p}{\varrho}\right]\right]\right) \\
& =\left(\alpha u U^{2}, \alpha v \gamma U^{2}, \alpha w \varepsilon U^{2}\right) .
\end{aligned}
$$

\subsection{Asymptotic assumptions and integral averages}

On the basis of physical assumptions, we assume that the vertical eddy viscosity is much larger than the horizontal one and that friction on the bottom depends linearly on the (relative) depth of the river, taken into account by the parameter $\varepsilon$. We can suppose that:

$$
\nu_{v}=\varepsilon \nu_{0}, \nu_{h}=O\left(\varepsilon^{2}\right), \alpha=\varepsilon \alpha_{0} .
$$

Rescaling (2.3) together with boundary conditions (2.12)-(2.14) and using (2.15)-(2.18), we obtain the following system:

$$
\left\{\begin{aligned}
\frac{\partial u}{\partial t}+ & \frac{\partial u^{2}}{\partial x}+\frac{\partial(u v)}{\partial y}+\frac{\partial(u w)}{\partial z}+\frac{1}{\varrho} \frac{\partial p}{\partial x} \lambda v+\frac{\partial}{\partial x}\left(2 \nu_{h} \frac{\partial u}{\partial x}\right)+\frac{\partial}{\partial y}\left(\frac{\nu_{h}}{\gamma^{2}} \frac{\partial u}{\partial y}+\nu_{h} \frac{\partial v}{\partial x}\right) \\
& +\frac{\partial}{\partial z}\left(\frac{\nu_{v}}{\varepsilon^{2}} \frac{\partial u}{\partial z}+\nu_{v} \frac{\partial w}{\partial x}\right), \\
\gamma^{2} & \left(\frac{\partial v}{\partial t}+\frac{\partial(u v)}{\partial x}+\frac{\partial v^{2}}{\partial y}+\frac{\partial(v w)}{\partial z}\right)+\frac{1}{\varrho} \frac{\partial p}{\partial y}-\lambda u+\frac{\partial}{\partial x}\left(\nu_{h} \frac{\partial u}{\partial y}+\nu_{h} \gamma^{2} \frac{\partial v}{\partial x}\right) \\
& +\frac{\partial}{\partial y}\left(2 \nu_{h} \frac{\partial v}{\partial y}\right)+\frac{\partial}{\partial z}\left(\frac{\nu_{v} \gamma^{2}}{\varepsilon^{2}} \frac{\partial v}{\partial z}+\nu_{v} \frac{\partial w}{\partial y}\right), \\
\varepsilon^{2} & \left(\frac{\partial w}{\partial t}+\frac{\partial(u w)}{\partial x}+\frac{\partial(v w)}{\partial y}+\frac{\partial w^{2}}{\partial z}\right)+\frac{1}{\varrho} \frac{\partial p}{\partial z}-G+\frac{\partial}{\partial x}\left(\nu_{v} \frac{\partial u}{\partial z}+\nu_{v} \varepsilon^{2} \frac{\partial w}{\partial x}\right) \\
& +\frac{\partial}{\partial y}\left(\nu_{v} \frac{\partial v}{\partial z}+\nu_{v} \frac{\varepsilon^{2}}{\gamma^{2}} \frac{\partial w}{\partial y}\right)+\frac{\partial}{\partial z}\left(2 \nu_{h} \frac{\partial w}{\partial z}\right), \\
\nabla & \cdot \boldsymbol{v}=0 .
\end{aligned}\right.
$$

The moduli of the rescaled normals are:

$$
N(\varepsilon, \eta)=\left[1+\varepsilon^{2}\left(\left(\frac{\partial \eta}{\partial x}\right)^{2}+\frac{1}{\gamma^{2}}\left(\frac{\partial \eta}{\partial y}\right)^{2}\right)\right]^{\frac{1}{2}}, \quad N(\varepsilon, b)=\left[1+\varepsilon^{2}\left(\left(\frac{\partial b}{\partial x}\right)^{2}+\frac{1}{\gamma^{2}}\left(\frac{\partial b}{\partial y}\right)^{2}\right)\right]^{\frac{1}{2}}
$$

together with boundary conditions on $\mathcal{S}$ and on $\mathcal{B}$ respectively. Therefore, indicating by $\left[\boldsymbol{\sigma}_{T_{11}}\right],\left[\boldsymbol{\sigma}_{T_{12}}\right],\left[\boldsymbol{\sigma}_{T_{22}}\right]$, $\left[\boldsymbol{\sigma}_{\boldsymbol{T}_{23}}\right],\left[\boldsymbol{\sigma}_{\boldsymbol{T} 33}\right]$ the rescaling of the stress tensor's components, we have on $\mathcal{S}$ :

$$
\left\{\begin{array}{l}
\frac{\partial \eta}{\partial x}\left(\frac{p}{\varrho}-2 \nu_{h} \frac{\partial u}{\partial x}\right)+\frac{\partial \eta}{\partial y}\left(-\frac{\nu_{h}}{\gamma^{2}} \frac{\partial u}{\partial y}-\nu_{h} \frac{\partial v}{\partial x}\right)+\frac{\nu_{v}}{\varepsilon^{2}} \frac{\partial u}{\partial z}+\nu_{v} \frac{\partial w}{\partial x}=-\boldsymbol{S}_{\boldsymbol{s u p} \mid x} N(\varepsilon, \eta), \\
\frac{\partial \eta}{\partial x}\left(-\nu_{h} \frac{\partial u}{\partial y}-\nu_{h} \gamma^{2} \frac{\partial v}{\partial x}\right)+\frac{\partial \eta}{\partial y}\left(\frac{p}{\varrho}-2 \nu_{h} \frac{\partial v}{\partial y}\right)+\nu_{v} \frac{\gamma^{2}}{\varepsilon^{2}} \frac{\partial v}{\partial z}+\nu_{v} \frac{\partial w}{\partial y}=-\boldsymbol{S}_{\boldsymbol{s u p} \mid y} N(\varepsilon, \eta) \\
-\frac{p}{\varrho}+2 \nu_{h} \frac{\partial w}{\partial z}=\varepsilon^{2}\left[\left(\frac{\partial \eta}{\partial x}\right)^{2}\left[\boldsymbol{\sigma}_{\boldsymbol{T} 11}\right]+\frac{2}{\gamma} \frac{\partial \eta}{\partial x} \frac{\partial \eta}{\partial y}\left[\boldsymbol{\sigma}_{\boldsymbol{T}_{12}}\right]+\frac{1}{\gamma^{2}}\left(\frac{\partial \eta}{\partial y}\right)^{2}\left[\boldsymbol{\sigma}_{\left.T_{22}\right]+}\right]\right. \\
\left.\quad-\frac{\partial \eta}{\partial x} \boldsymbol{S}_{\boldsymbol{s u p} \mid x} N(\varepsilon, \eta)-\frac{1}{\gamma^{2}} \frac{\partial \eta}{\partial y} \boldsymbol{S}_{\boldsymbol{s u p} \mid y} N(\varepsilon, \eta)\right]-p_{a} N(\varepsilon, \eta), \\
w=\frac{\partial \eta}{\partial t}+u \frac{\partial \eta}{\partial x}+v \frac{\partial \eta}{\partial y}
\end{array}\right.
$$


Note that the third equation above is the result of a linear combination of the three equations representing the dynamical condition on the free surface (see (2.12)).

Following the same step, we rescale the conditions on $\mathcal{B}$ as follows:

$$
\left\{\begin{array}{l}
\frac{\partial b}{\partial x}\left(-\frac{p}{\varrho}+2 \nu_{h} \frac{\partial u}{\partial x}\right)+\frac{\partial b}{\partial y}\left(\frac{\nu_{h}}{\gamma^{2}} \frac{\partial u}{\partial y}+\nu_{h} \frac{\partial v}{\partial x}\right)-\frac{\nu_{v}}{\varepsilon^{2}} \frac{\partial u}{\partial z}-\nu_{v} \frac{\partial w}{\partial x}=-\alpha_{0} u N(\varepsilon, b) \\
\frac{\partial b}{\partial x}\left(\nu_{h} \frac{\partial u}{\partial y}+\nu_{h} \gamma^{2} \frac{\partial v}{\partial x}\right)+\frac{\partial b}{\partial y}\left(-\frac{p}{\varrho}+2 \nu_{h} \frac{\partial v}{\partial y}\right)-\nu_{v} \frac{\gamma^{2}}{\varepsilon^{2}} \frac{\partial v}{\partial z}-\nu_{v} \frac{\partial w}{\partial y}=-\alpha_{0} \gamma^{2} v N(\varepsilon, b) \\
-\frac{p}{\varrho}+2 \nu_{h} \frac{\partial w}{\partial z}=\varepsilon^{2}\left[\left(\frac{\partial b}{\partial x}\right)^{2}\left[\boldsymbol{\sigma}_{T 11}\right]+\frac{2}{\gamma} \frac{\partial b}{\partial x} \frac{\partial b}{\partial y}\left[\boldsymbol{\sigma}_{T 12}\right]+\frac{1}{\gamma^{2}}\left(\frac{\partial b}{\partial y}\right)^{2}\left[\boldsymbol{\sigma}_{T 22}\right]\right. \\
\left.+\frac{\partial b}{\partial x} \alpha_{0} u N(\varepsilon, b)+\frac{\partial b}{\partial y} \alpha_{0} v \gamma N(\varepsilon, b)+\alpha_{0} w N(\varepsilon, b)\right] \\
w=u \frac{\partial b}{\partial x}+v \frac{\partial b}{\partial y}
\end{array}\right.
$$

Now, the idea is to neglect quantities which are $O\left(\varepsilon^{2}\right)$ in $(2.20)-(2.22)$. In view of such approximations, we may then rewrite $(2.20)$ as follows:

$$
\left\{\begin{array}{l}
\frac{\partial u}{\partial t}+\frac{\partial u^{2}}{\partial x}+\frac{\partial(u v)}{\partial y}+\frac{\partial(u w)}{\partial z}+\frac{1}{\varrho} \frac{\partial p}{\partial x}=\lambda v+\frac{\partial}{\partial z}\left(\frac{\nu_{v}}{\varepsilon^{2}} \frac{\partial u}{\partial z}+\nu_{v} \frac{\partial w}{\partial x}\right) \\
\gamma^{2}\left(\frac{\partial v}{\partial t}+\frac{\partial(u v)}{\partial x}+\frac{\partial v^{2}}{\partial y}+\frac{\partial(v w)}{\partial z}\right)+\frac{1}{\varrho} \frac{\partial p}{\partial y}-\lambda u+\frac{\partial}{\partial z}\left(\frac{\nu_{v} \gamma^{2}}{\varepsilon^{2}} \frac{\partial v}{\partial z}+\nu_{v} \frac{\partial w}{\partial y}\right) \\
\frac{1}{\varrho} \frac{\partial p}{\partial z}=-G+\frac{\partial}{\partial x}\left(\nu_{v} \frac{\partial u}{\partial z}\right)+\frac{\partial}{\partial y}\left(\nu_{v} \frac{\partial v}{\partial z}\right), \\
\nabla \cdot \boldsymbol{v}=0 .
\end{array}\right.
$$

Moreover, as soon as the gradient of the free surface remains bounded and recalling that the bathymetry surface is assumed to be regular on $\bar{\Omega}$, we have that:

$$
N(\varepsilon, \eta)=1+O\left(\varepsilon^{2}\right), \quad N(\varepsilon, b)=1+O\left(\varepsilon^{2}\right) .
$$

We then complete (2.23) with upper and lower boundary conditions:

$$
\begin{array}{ll}
\text { on } \mathcal{S}: \quad & \left\{\begin{array}{l}
\frac{\partial \eta}{\partial x} \frac{p}{\varrho}+\frac{\nu_{v}}{\varepsilon^{2}} \frac{\partial u}{\partial z}+\nu_{v} \frac{\partial w}{\partial x}=-\boldsymbol{S}_{\mathbf{s u p} \mid x}, \\
\frac{\partial \eta}{\partial y} \frac{p}{\varrho}+\nu_{v} \frac{\gamma^{2}}{\varepsilon^{2}} \frac{\partial v}{\partial z}+\nu_{v} \frac{\partial w}{\partial y}=-\boldsymbol{S}_{\mathbf{s u p} \mid y} \\
\frac{p}{\varrho}=p_{a}, \\
w=\frac{\partial \eta}{\partial t}+u \frac{\partial \eta}{\partial x}+v \frac{\partial \eta}{\partial y},
\end{array}\right. \\
\text { on } \mathcal{B}: \quad\left\{\begin{array}{l}
\frac{\partial b}{\partial x} \frac{p}{\varrho}+\frac{\nu_{v}}{\varepsilon^{2}} \frac{\partial u}{\partial z}+\nu_{v} \frac{\partial w}{\partial x}=\alpha_{0} u \\
\frac{\partial b}{\partial y} \frac{p}{\varrho}+\nu_{v} \frac{\gamma^{2}}{\varepsilon^{2}} \frac{\partial v}{\partial z}+\nu_{v} \frac{\partial w}{\partial y}=\alpha_{0} \gamma^{2} v \\
\frac{p}{\varrho}=0, \\
w=u \frac{\partial b}{\partial x}+v \frac{\partial b}{\partial y}
\end{array}\right.
\end{array}
$$

The initial condition and the boundary conditions on $(0, T) \times \boldsymbol{\Gamma}$ are not affected by the rescaling. 
As we suppose the vertical scale to be "small enough" with respect to the longitudinal scale, we don't loose much in precision (in the following we will specify exactly how much) if in (2.23) we substitute to the velocity components and to the pressure, the corresponding integral averages on the vertical axis from $z=b(x, y),(x, y) \in$ $\bar{\Omega}$ to $z=\eta(t, x, y),(t, x, y) \in[0, T] \times \bar{\Omega}$. Recalling that $H=\eta-b$ and using the notation

$$
\bar{f}(t, x, y)=\frac{1}{H(t, x, y)} \int_{b(x, y)}^{\eta(t, x, y)} f(t, x, y, z) \mathrm{d} z,
$$

if in (2.23) we integrate the first two equations from the bottom to the free surface and the third equation from the bottom to a given vertical level $z$, we obtain on $(0, T] \times \Omega($ using $(2.24)$ and $(2.25))$ :

$$
\left\{\begin{array}{l}
\frac{\partial}{\partial t}(H \bar{u})+\frac{\partial}{\partial x}\left(H \overline{u^{2}}\right)+\frac{\partial}{\partial y}(H \overline{u v})+\frac{\partial}{\partial x}\left(H \frac{1}{\varrho} \bar{p}\right)=\lambda H \bar{v}-\boldsymbol{S}_{\mathbf{s u p} \mid x}-\alpha_{0} u(t, x, y, b) \\
\gamma^{2}\left[\frac{\partial}{\partial t}(H \bar{v})+\frac{\partial}{\partial x}(H \overline{u v})+\frac{\partial}{\partial y}\left(H \overline{v^{2}}\right)\right]+\frac{\partial}{\partial y}\left(H \frac{1}{\varrho} \bar{p}\right)=-\lambda H \bar{u}-\boldsymbol{S}_{\mathbf{s u p} \mid y}-\gamma^{2} \alpha_{0} v(t, x, y, b) \\
\frac{1}{\varrho} p(t, x, y, z)=p_{a}+G(\eta(t, x, y)-z)+\nu_{v} \frac{\partial u}{\partial x}(t, x, y, \eta)-\nu_{v} \frac{\partial u}{\partial x}(t, x, y, z) \\
\quad+\nu_{v} \frac{\partial v}{\partial y}(t, x, y, \eta)-\nu_{v} \frac{\partial v}{\partial y}(t, x, y, z) \\
\frac{\partial H}{\partial t}+\frac{\partial}{\partial x}(H \bar{u})+\frac{\partial}{\partial y}(H \bar{v})=0
\end{array}\right.
$$

Recalling that normals to $\partial \Omega$ (see Fig. 2) don't depend on $z$, and assuming $\overline{\boldsymbol{v}}=(\bar{u}, \bar{v})^{T}$, the boundary conditions are now set on $(0, T) \times \Gamma$ by:

$$
\left\{\begin{array}{l}
\overline{\boldsymbol{v}} \cdot \boldsymbol{n}_{ \pm}= \pm \bar{v}(t, x, y)=0 \quad \text { on }(0, T) \times \boldsymbol{\Gamma}_{\boldsymbol{c}} \\
\boldsymbol{n}_{\text {in }} \cdot \overline{\boldsymbol{v}}(t, x, y)=\bar{u}(t, x, y)>0 \quad \text { on }(0, T) \times \boldsymbol{\Gamma}_{\text {in }} \\
\boldsymbol{n}_{\text {out }} \cdot \overline{\boldsymbol{v}}(t, x, y)=-\bar{u}(t, x, y)<0 \quad \text { on }(0, T) \times \boldsymbol{\Gamma}_{\text {out }}
\end{array}\right.
$$

Initial conditions on $\{t=0\} \times \Omega$ are given by:

$$
\overline{\boldsymbol{v}}=\overline{\boldsymbol{v}_{0}}, \quad H=H_{0} .
$$

Let us now consider terms with an approximation of $O(\varepsilon)$. From (2.23)-(2.25) and (2.19), one obtains:

Therefore:

$$
\begin{gathered}
\frac{1}{\varrho} p(t, x, y, z)=\boldsymbol{S}_{\sup \mid z}+G(\eta(t, x, y)-z)+O(\varepsilon) \\
\frac{\partial^{2} u}{\partial z^{2}}(t, x, y, z)=O(\varepsilon), \quad \frac{\partial u}{\partial z}(t, x, y, b)=O(\varepsilon), \quad \frac{\partial u}{\partial z}(t, x, y, \eta)=O(\varepsilon) \\
\frac{\partial^{2} v}{\partial z^{2}}(t, x, y, z)=O(\varepsilon), \quad \frac{\partial v}{\partial z}(t, x, y, b)=O(\varepsilon), \quad \frac{\partial v}{\partial z}(t, x, y, \eta)=O(\varepsilon) .
\end{gathered}
$$

and straightforward we conclude that

$$
u(t, x, y, z)=\bar{u}(t, x, y)+O(\varepsilon), \quad v(t, x, y, z)=\bar{v}(t, x, y)+O(\varepsilon),
$$

$$
\overline{u^{2}}(t, x, y)=\bar{u}^{2}(t, x, y)+O\left(\varepsilon^{2}\right), \quad \overline{v^{2}}(t, x, y)=\bar{v}^{2}(t, x, y)+O\left(\varepsilon^{2}\right), \quad \overline{u v}=\bar{u} \bar{v}+O\left(\varepsilon^{2}\right) .
$$

In order to derive our model, some further restrictions on given data have to be taken into account. Firstly, we assume that

$$
\nabla b=O(\varepsilon), \quad \nabla p_{a}=O(\varepsilon)
$$


that is, we are supposing a slow varying bathymetry and a small atmospheric pressure gradient. Moreover, as in [23], we suppose the horizontal components of the velocity to admit a linear asymptotic expansion to the second order with respect to $\varepsilon$ as:

$$
u=\bar{u}+\varepsilon u_{1}+O\left(\varepsilon^{2}\right), \quad v=\bar{v}+\varepsilon v_{1}+O\left(\varepsilon^{2}\right),
$$

where the zero order term is given by the mean value of quantities $u$ and $v$ respectively, computed as in (2.26). This allows us to deduce from (2.32) that:

$$
\frac{\partial u}{\partial \varphi}=\frac{\partial \bar{u}}{\partial \varphi}+O(\varepsilon), \quad \frac{\partial v}{\partial \varphi}=\frac{\partial \bar{v}}{\partial \varphi}+O(\varepsilon) \quad \text { where } \quad \varphi \in\{t, x, y\}
$$

As in [7], Proposition 4.1, we obtain for the velocity and for the pressure the following approximations to the second order with respect to $\varepsilon$ :

$$
\begin{array}{rr}
u(t, x, y, b)=\frac{\bar{u}(t, x, y)}{1+\frac{\alpha_{0} \varepsilon H}{3 \nu_{0}}}+O\left(\varepsilon^{2}\right), & v(t, x, y, b)=\frac{\bar{v}(t, x, y)}{1+\frac{\alpha_{0} \varepsilon H}{3 \nu_{0} \gamma^{2}}}+O\left(\varepsilon^{2}\right) \\
\frac{\partial}{\partial x}\left(H \frac{1}{\varrho} \bar{p}\right)=\frac{\partial\left(H p_{a}\right)}{\partial x}+\frac{G}{2} \frac{\partial H^{2}}{\partial x}+O\left(\varepsilon^{2}\right), & \frac{\partial}{\partial y}\left(H \frac{1}{\varrho} \bar{p}\right)=\frac{\partial\left(H p_{a}\right)}{\partial y}+\frac{G}{2} \frac{\partial H^{2}}{\partial y}+O\left(\varepsilon^{2}\right) .
\end{array}
$$

Substituting (2.32) and (2.33) in $(2.27)$, one obtains on $(0, T) \times \Omega$, with a precision of $O\left(\varepsilon^{2}\right)$ :

$$
\left\{\begin{array}{l}
\frac{\partial(H \bar{u})}{\partial t}+\frac{\partial\left(H \bar{u}^{2}\right)}{\partial x}+\frac{\partial(H \bar{u} \bar{v})}{\partial y}+\frac{G}{2} \frac{\partial H^{2}}{\partial x}= \\
\lambda H \bar{v}-\frac{\partial\left(H p_{a}\right)}{\partial x}-\boldsymbol{S}_{\sup \mid x}-\frac{\alpha_{0}}{1+\frac{\alpha_{0} \varepsilon H}{3 \nu_{0}}} \bar{u} \\
\gamma^{2}\left(\frac{\partial(H \bar{v})}{\partial t}+\frac{\partial(H \bar{u} \bar{v})}{\partial x}+\frac{\partial\left(H \bar{v}^{2}\right)}{\partial y}\right)+\frac{G}{2} \frac{\partial H^{2}}{\partial y}= \\
-\lambda H \bar{u}-\frac{\partial\left(H p_{a}\right)}{\partial y}-\boldsymbol{S}_{\sup \mid y}-\frac{\alpha_{0} \gamma^{2}}{1+\frac{\alpha_{0} \varepsilon H}{3 \nu_{0} \gamma^{2}}} \bar{v} \\
\frac{\partial H}{\partial t}+\frac{\partial}{\partial x}(H \bar{u})+\frac{\partial}{\partial y}(H \bar{v})=0
\end{array}\right.
$$

Recovering dimensions in $(2.38)$ we have on $(0, T) \times \Omega$ :

$$
\left\{\begin{array}{c}
\frac{\partial(H \bar{u})}{\partial t}+\frac{\partial\left(H \bar{u}^{2}\right)}{\partial x}+\frac{\partial(H \bar{u} \bar{v})}{\partial y}+\frac{g}{2} \frac{\partial H^{2}}{\partial x}= \\
l H \bar{v}-\frac{\partial\left(H p_{a}\right)}{\partial x}-s_{\sup \mid x}-\frac{k}{1+\frac{k H}{3 \mu_{v}}} \bar{u} \\
\frac{\partial(H \bar{v})}{\partial t}+\frac{\partial(H \bar{u} \bar{v})}{\partial x}+\frac{\partial\left(H \bar{v}^{2}\right)}{\partial y}+\frac{g}{2} \frac{\partial H^{2}}{\partial y}= \\
-l H \bar{u}-\frac{\partial\left(H p_{a}\right)}{\partial y}-s_{\mathbf{s u p}_{\mid y}}-\frac{k}{1+\frac{k H}{3 \mu_{v}}} \bar{v} \\
\frac{\partial H}{\partial t}+\frac{\partial}{\partial x}(H \bar{u})+\frac{\partial}{\partial y}(H \bar{v})=0
\end{array}\right.
$$


which in compact form reads as:

$$
\left\{\begin{array}{l}
\frac{\partial}{\partial t}(H \overline{\boldsymbol{v}})+\nabla \cdot(H \overline{\boldsymbol{v}} \otimes \overline{\boldsymbol{v}})+g H \nabla H=l H \hat{\hat{\boldsymbol{v}}}-\nabla\left(H p_{a}\right)-\boldsymbol{s}_{\sup \mid x y}-\frac{k}{1+\frac{k H}{3 \mu_{v}}} \overline{\boldsymbol{v}} \\
\frac{\partial H}{\partial t}+\nabla \cdot(H \overline{\boldsymbol{v}})=0 .
\end{array}\right.
$$

Gradient and divergence operators are meant here as two-dimensional operators, $\left.\boldsymbol{s}_{\boldsymbol{s u p}}\right|_{x, y}$ represents the vector of the first two components of the surface stress, $\boldsymbol{v}$ is the two-dimensional vector of the two horizontal velocity components $(\boldsymbol{v}=(u, v))$, while through vector $\hat{\hat{\boldsymbol{v}}}=(\bar{v},-\bar{u})$ we represent the Coriolis term.

We refer to (2.40) as our SW2d model, completed by boundary and initial conditions (2.28), (2.29).

System (2.40) has now become two-dimensional, meaning that spatial variables involved are only two: $x$ and $y$; the same way, only two averaged velocity components are involved: $\bar{u}$ and $\bar{v}$. Notice further, that the number of momentum conservation equations has been reduced from three (in (2.3)) to only two.

Remark that although we are supposing the bottom surface is slow varying (see (2.34)), in our model (2.39) we don't have any term like $\nabla b$. In fact all the terms connected to the bottom topography varying are canceled through averaging NS3d equations, taking boundary conditions on the bottom surface into account.

Remark 2.1. Let us recall the classical inviscid two-dimensional Shallow Water system (see, for instance, [1]) on $\Omega \times(0, T]$ :

$$
\left\{\begin{array}{l}
\frac{\partial}{\partial t}(H \overline{\boldsymbol{v}})+\nabla \cdot(H \overline{\boldsymbol{v}} \otimes \overline{\boldsymbol{v}})+g H \nabla H=l H \hat{\boldsymbol{v}}-H \nabla p_{a}+\boldsymbol{s}_{\mathbf{s u p} \mid x y}-g|\overline{\boldsymbol{v}}| \frac{\overline{\boldsymbol{v}}}{C_{1}^{2}} \\
\frac{\partial H}{\partial t}+\nabla \cdot(H \overline{\boldsymbol{v}})=0
\end{array}\right.
$$

where we are considering notation (2.7). The friction term is modeled through the so-called Chézy formula which involves a suitable proportion constant $C_{1}$.

Notice, in comparison with our model (2.40), that classical model presents a different treatment of the friction term and of the surface stresses, while convective and pressure terms are left the same.

\subsection{Recovering 3d-like informations}

Thanks to the continuity equation in (2.23), recalling the last relation in (2.25), we recover the vertical component of the velocity by the relation:

$$
\begin{aligned}
w(t, x, y, z)=u(t, x, y, b(x, y)) \frac{\partial b}{\partial x}(x, y)+v(t, x, y, & b(x, y)) \frac{\partial b}{\partial y}(x, y)+ \\
& -\int_{b(x, y)}^{z} \frac{\partial u}{\partial x}(t, x, y, r) \mathrm{d} r-\int_{b(x, y)}^{z} \frac{\partial v}{\partial y}(t, x, y, r) \quad \mathrm{d} r .
\end{aligned}
$$

Moreover, by (2.32), (2.42) takes the form

$$
\begin{aligned}
w(t, x, y, z)=\bar{u}(t, x, y) \frac{\partial b}{\partial x}(x, y)+\bar{v}(t, x, y) \frac{\partial b}{\partial y}(x, y)-(z-b(x, y)) \frac{\partial \bar{u}}{\partial x}(t, x, y) & \\
& -(z-b(x, y)) \frac{\partial \bar{v}}{\partial y}(t, x, y)+O(\varepsilon) .
\end{aligned}
$$


Then, we calculate the average vertical velocity as

$$
\bar{w}(t, x, y)=\bar{u}(t, x, y) \frac{\partial b}{\partial x}(x, y)+\bar{v}(t, x, y) \frac{\partial b}{\partial y}(x, y)-\frac{H}{2}(t, x, y) \frac{\partial \bar{u}}{\partial x}(t, x, y)-\frac{H}{2}(t, x, y) \frac{\partial \bar{v}}{\partial y}(t, x, y)+O(\varepsilon) .
$$

It is important to notice that we can obtain also the average value of the total stress tensor (2.6) with an approximation of $O\left(\varepsilon^{2}\right)$. In fact, if we consider the rescaling of the total stress tensor (2.6), using the notation in $(2.15)-(2.18)$, we have:

$$
\left[\left[\frac{\boldsymbol{\sigma}_{\boldsymbol{T}}}{\varrho}\right]\right]=U^{2} \cdot\left[\begin{array}{ccc}
-\frac{p}{\varrho}+2 \nu_{h} \frac{\partial u}{\partial x} & \frac{\nu_{h}}{\gamma} \frac{\partial u}{\partial y}+\nu_{h} \gamma \frac{\partial v}{\partial x} & \frac{\nu_{v}}{\varepsilon} \frac{\partial u}{\partial z}+\nu_{v} \varepsilon \frac{\partial w}{\partial x} \\
\frac{\nu_{h}}{\gamma} \frac{\partial u}{\partial y}+\nu_{h} \gamma \frac{\partial v}{\partial x} & -\frac{p}{\varrho}+2 \nu_{h} \frac{\partial v}{\partial y} & \nu_{v} \frac{\gamma}{\varepsilon} \frac{\partial v}{\partial z}+\nu_{v} \frac{\varepsilon}{\gamma} \frac{\partial w}{\partial y} \\
\frac{\nu_{v}}{\varepsilon} \frac{\partial u}{\partial z}+\nu_{v} \varepsilon \frac{\partial w}{\partial x} & \nu_{v} \frac{\gamma}{\varepsilon} \frac{\partial v}{\partial z}+\nu_{v} \frac{\varepsilon}{\gamma} \frac{\partial w}{\partial y} & -\frac{p}{\varrho}+2 \nu_{h} \frac{\partial w}{\partial z}
\end{array}\right] .
$$

Then, approximating to $O\left(\varepsilon^{2}\right)$, using (2.43), (2.32) and (2.30) we conclude that

$$
\left[\left[\frac{\boldsymbol{\sigma}_{\boldsymbol{T}}}{\varrho}\right]\right]-U^{2}\left(p_{a}+G(\eta(t, x, y)-z)\right) \boldsymbol{I}_{3 \times 3}
$$

Averaging and recovering dimensions, we obtain, modulus an $O\left(\varepsilon^{2}\right)$ :

$$
\frac{\overline{\boldsymbol{\sigma}_{\boldsymbol{T}}}}{\varrho}=-\left(p_{a}+g \frac{H}{2}\right) \boldsymbol{I}_{3 \times 3} .
$$

Remark that only pressure terms don't drop through the $O\left(\varepsilon^{2}\right)$ approximation.

\subsection{Further remarks}

Let us observe that if we average model (2.38) along $y$-axis (where $y \in(-L / 2, L / 2)$ ) then it is not possible anymore to extend the asymptotic reasoning described in the last paragraph to obtain a one-dimensional model from the two-dimensional SW2d model (2.40).

It is important to notice that the whole asymptotic analysis previously described starts from the validity of (2.31), but a relation similar to (2.31) like:

$$
\frac{\partial^{2} u}{\partial y^{2}}(t, x, y, z)=O(\varepsilon), \frac{\partial^{2} v}{\partial y^{2}}(t, x, y, z)=O(\varepsilon)
$$

does not hold as one can verify from (2.23). In fact, in sight of (2.19), it is evident that terms $\frac{\partial^{2} u}{\partial z^{2}}$ and $\frac{\partial^{2} v}{\partial z^{2}}$ appear, respectively, in the first and in the second equation of (2.23) with an $\varepsilon$ factor at the denominator, which permits $(2.31)$ to be valid. On the other side, it can be seen that this fact doesn't hold for terms like $\frac{\partial^{2} u}{\partial y^{2}}$ and $\frac{\partial^{2} v}{\partial y^{2}}$.

Remark that an analogous derivation of our model (2.40) together with boundary and initial conditions (2.28), (2.29) can be obtained with a general bounded domain with a piecewise smooth boundary, provided boundary conditions are of the form $\overline{\boldsymbol{v}} \cdot \boldsymbol{n}_{\leq}^{\gtrless}$, where $\boldsymbol{n}$ doesn't depend on $z$, or of Dirichlet type. 


\section{The Symmetrized MOdel (SSW2D)}

Let us consider again the SW2d system (2.39) in conservative form, denoting, for the sake of simplicity, $\bar{u}$, $\bar{v}$ and $\mu_{v}$, respectively with $u, v$ and $\mu$ on $(0, T) \times \Omega$. This system is completed with initial and boundary conditions $(2.28),(2.29)$. In quasi-linear form the system $(2.39)$ can be rewritten in the form:

$$
\frac{\partial \boldsymbol{V}}{\partial t}+A_{1}(t, x, y, \boldsymbol{V}) \frac{\partial \boldsymbol{V}}{\partial x}+A_{2}(t, x, y, \boldsymbol{V}) \frac{\partial \boldsymbol{V}}{\partial y}=G(t, x, y, \boldsymbol{V}),
$$

where $\boldsymbol{V}=(H u, H v, H)^{T}$,

$$
\begin{aligned}
& A_{1}(t, x, y, \boldsymbol{V})=\left[\begin{array}{ccc}
2 u & 0 & g H+p_{a}-u^{2} \\
v & u & -u v \\
1 & 0 & 0
\end{array}\right], \\
& A_{2}(t, x, y, \boldsymbol{V})=\left[\begin{array}{ccc}
v & u & -u v \\
0 & 2 v & g H+p_{a}-v^{2} \\
0 & 1 & 0
\end{array}\right],
\end{aligned}
$$

and

$$
G(t, x, y, \boldsymbol{V})=\left(\begin{array}{c}
l H v-H \frac{\partial p_{a}}{\partial x}-s_{\sup _{\mid x}}-\frac{k u}{1+\frac{k H}{3 \mu}} \\
-l H u-H \frac{\partial p_{a}}{\partial y}-s_{\sup \mid y}-\frac{v}{1+\frac{k H}{3 \mu}} \\
0
\end{array}\right)
$$

Remark that the dependence of $A_{1}, A_{2}$ and $G$ on $t, x$ and $y$, is due to the explicit dependence of terms $p_{a}$ and $s_{\text {sup } \mid x y}$ on those variables.

The following proposition holds:

Proposition 3.1. Consider:

$$
A(\boldsymbol{\xi}, t, x, y, \boldsymbol{V})=\xi_{1} A_{1}(t, x, y, \boldsymbol{V})+\xi_{2} A_{2}(t, x, y, \boldsymbol{V}), \quad \forall \boldsymbol{\xi} \in \mathbb{R}^{2} \backslash\{0\}
$$

where $A_{1}$ and $A_{2}$ are given by (3.2). The three eigenvalues of the SW2d system are then given by:

$$
\lambda_{2}(\boldsymbol{\xi}, t, x, y, \boldsymbol{V})=\xi_{1} u+\xi_{2} v, \quad \lambda_{1,3}(\boldsymbol{\xi}, t, x, y, \boldsymbol{V})=\xi_{1} u+\xi_{2} v \mp \sqrt{g H+p_{a}} \sqrt{\xi_{1}^{2}+\xi_{2}^{2}}
$$

Therefore, the SW2d system is a (first order-quasilinear) strictly hyperbolic system.

In fact, since $p_{a}$ is a positive constant (as atmospheric pressure is always positive), we have that:

$$
\lambda_{1}(\boldsymbol{\xi}, t, x, y, \boldsymbol{V})<\lambda_{2}(\boldsymbol{\xi}, t, x, y, \boldsymbol{V})<\lambda_{3}(\boldsymbol{\xi}, t, x, y, \boldsymbol{V}) .
$$

Then, following the definition cited in Serre [22] (Vol. I, Chap. 3), the model is (quasilinear) hyperbolic.

A wide analytical theory on hyperbolic problems has been developed in the case they are symmetrizable. Therefore, we will first consider a symmetrization of (first order hyperbolic) SW2d system, given by (3.1), in order to obtain the symmetric system, denoted by SSW2d, which we show to be hyperbolic as well. We will then exploit if SSW2d model may be considered as the inviscid limit of a sequence of artificial viscous perturbed (second order) parabolic problems.

We concentrate our attention on the case when $p_{a}$ is a non-null function of $x, y$ and $t$. 


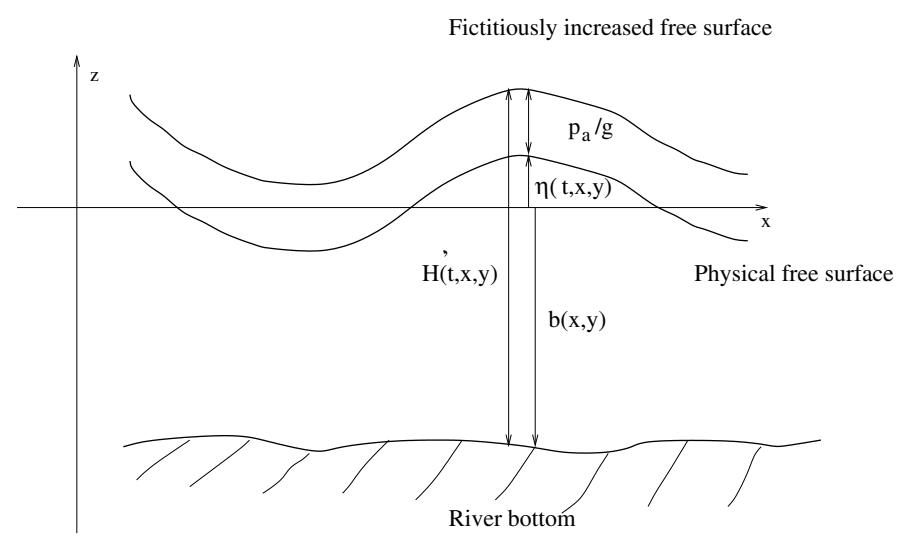

FiguRE 3. Vertical cross-section with elevation "fictitiously increased".

Physical Postulate 1. A Shallow Water flow on which atmospheric pressure is acting, behaves equivalently (from a physical point of view) to a Shallow Water flow without atmospheric pressure, in which the elevation is "fictitiously" increased by the constant value $p_{a} / g$.

Let us explain in detail what we mean as a Shallow Water flow without atmospheric pressure, in which the elevation is "fictitiously" increased by the constant value $p_{a} / g$, going through SW2d model derivation exposed in Section 2.

We consider our incompressible fluid confined into a three dimensional domain $\mathcal{U}^{\prime}$ which is vertically bounded by the surfaces $z=\eta^{\prime}(\boldsymbol{x}, t)$, where $\eta^{\prime}=\eta+p_{a} / g$ and $z=b(\boldsymbol{x})$, so that (2.1) becomes:

$$
\mathcal{U}^{\prime}:=\left\{(t, x, y, z): t \in(0, T),(x, y) \in \Omega, z \in\left(b(x, y), \eta^{\prime}(t, x, y)\right)\right\}
$$

denoting again by $\Omega$ the domain introduced in $(2.2)$, where $T>0$, we have that $\eta^{\prime}:[0, T] \times \bar{\Omega} \rightarrow \mathbb{R}(T>0)$ is the "fictitious" elevation (see Fig. 3), while $b: \bar{\Omega} \rightarrow \mathbb{R}^{-}$is as before the bottom depth with respect to the same reference level; $H^{\prime}$, given by:

$$
H^{\prime}=\eta^{\prime}-b=H+\frac{p_{a}}{g}
$$

is always strictly positive (as $\frac{p_{a}}{g}$ ) and represents the total height of the fluid from the bottom to the "fictitious" free surface. Let $\mathcal{S}^{\prime}$ be the free surface connected to the domain $\mathcal{U}^{\prime}$, let $\mathcal{B}$ be the bottom surface and $\mathcal{L}^{\prime}$ be the lateral surface given by a formula analogous to (2.11). Let us consider again NS3d equations (2.3) with stress tensor assigned by (2.5) and total stress tensor assigned by (2.6). Now, the surface stress tensor is given by $s_{\text {sup }}^{\prime}$ so that (2.7) becomes:

$$
s_{\sup _{\mid x y}}^{\prime}=C|W| W, \quad s_{\text {sup }_{\mid z}}^{\prime}=0
$$

in fact the horizontal components of the surface stress tensor (wind effect) are left unaltered, while we are neglecting the effect of the atmospheric pressure as the vertical component of the surface stress (we are recovering this effect adding the fictitious increment $\frac{p_{a}}{g}$ to the elevation $H$ ). We then consider boundary conditions analogous to $(2.12,2.13)$ and $(2.14)$ and perform adimensionalizations, asymptotic assumptions and integral averages as in Sections 2.1 and 2.4. Assumption (2.34) on the "smallness" of $\nabla p_{a}$ becomes now not restrictive as we are supposing that no atmospheric pressure is acting as the vertical component of the surface stress tensor. 
We therefore obtain on $(0, T) \times \Omega$ the system:

$$
\left\{\begin{array}{l}
\frac{\partial\left(H^{\prime} u\right)}{\partial t}+\frac{\partial\left(H^{\prime} u^{2}\right)}{\partial x}+\frac{\partial\left(H^{\prime} u v\right)}{\partial y}+\frac{g}{2} \frac{\partial H^{\prime 2}}{\partial x} l H^{\prime} v-s_{\mathbf{s u p}_{\mid x}}-\frac{k}{1+\frac{k H^{\prime}}{3 \mu}} u \\
\frac{\partial\left(H^{\prime} v\right)}{\partial t}+\frac{\partial\left(H^{\prime} u v\right)}{\partial x}+\frac{\partial\left(H^{\prime} v^{2}\right)}{\partial y}+\frac{g}{2} \frac{\partial H^{\prime 2}}{\partial y}-l H^{\prime} u-s_{\mathbf{s u p}_{\mid y}}-\frac{k}{1+\frac{k H^{\prime}}{3 \mu}} v \\
\frac{\partial H^{\prime}}{\partial t}+\frac{\partial}{\partial x}\left(H^{\prime} u\right)+\frac{\partial}{\partial y}\left(H^{\prime} v\right)=0
\end{array}\right.
$$

In quasi-linear form system (3.7) reads as:

$$
\frac{\partial \hat{\boldsymbol{V}}}{\partial t}+\hat{A}_{1}(\hat{\boldsymbol{V}}) \frac{\partial \hat{\boldsymbol{V}}}{\partial x}+\hat{A}_{2}(\hat{\boldsymbol{V}}) \frac{\partial \hat{\boldsymbol{V}}}{\partial y}=\hat{G}(t, x, y, \hat{\boldsymbol{V}})
$$

where we posed $\hat{\boldsymbol{V}}=\left(H^{\prime} u, H^{\prime} v, H^{\prime}\right)^{T}$,

$$
\hat{A}_{1}(\hat{\boldsymbol{V}})=\left[\begin{array}{ccc}
2 u & 0 & g H^{\prime}-u^{2} \\
v & u & -u v \\
1 & 0 & 0
\end{array}\right], \quad \hat{A}_{2}(\hat{\boldsymbol{V}})=\left[\begin{array}{ccc}
v & u & -u v \\
0 & 2 v & g H^{\prime}-v^{2} \\
0 & 1 & 0
\end{array}\right]
$$

and

$$
\hat{G}(t, x, y, \hat{\boldsymbol{V}})=\left(\begin{array}{c}
l H^{\prime} v-s_{\mathbf{s u p}_{\mid x}}-\frac{k u}{1+\frac{k H^{\prime}}{3 \mu}} \\
-l H^{\prime} u-s_{\mathbf{s u p}_{\mid y}}-\frac{k v}{1+\frac{k H^{\prime}}{3 \mu}} \\
0
\end{array}\right)
$$

Remark 3.1. We underline that system (3.1) may not be recovered by taking relation (3.5) into account and substituting into (3.7).

Now we can symmetrize system (3.8) i.e., performing a suitable change of variables we transform matrices $\hat{A}_{1}$ and $\hat{A}_{2}$ into symmetric ones (the right hand side $\hat{G}$ will be consequently changed).

Let us introduce the new vector variable:

$$
\boldsymbol{U}=\left(u, v, H_{g}\right)^{T}, \quad H_{g}=2 \sqrt{g H^{\prime}}=2 \sqrt{g H+p_{a}}
$$

where $H_{g}$, known as the celerity variable, is always positive even if the effective elevation $H$ is vanishing. In fact we have:

$$
H_{g} \geq 2 \sqrt{p_{a}}>0
$$

Using the new set of variables, we can rewrite system (3.8) in the following form

$$
\frac{\partial \hat{\boldsymbol{V}}}{\partial \boldsymbol{U}} \frac{\partial \boldsymbol{U}}{\partial t}+\hat{A}_{1}(\hat{\boldsymbol{V}}) \frac{\partial \hat{\boldsymbol{V}}}{\partial \boldsymbol{U}} \frac{\partial \boldsymbol{U}}{\partial x}+\hat{A}_{2}(\hat{\boldsymbol{V}}) \frac{\partial \hat{\boldsymbol{V}}}{\partial \boldsymbol{U}} \frac{\partial \boldsymbol{U}}{\partial y}=\hat{G}(t, x, y, \hat{\boldsymbol{V}})
$$


We denote

$$
\hat{A}_{0}(\hat{\boldsymbol{V}}) \equiv \frac{\partial \hat{\boldsymbol{V}}}{\partial \boldsymbol{U}}\left[\begin{array}{ccc}
H^{\prime} & 0 & \frac{H^{\prime} u}{\sqrt{g H^{\prime}}} \\
0 & H^{\prime} & \frac{H^{\prime} v}{\sqrt{g H^{\prime}}} \\
0 & 0 & \frac{H^{\prime}}{\sqrt{g H^{\prime}}}
\end{array}\right], \quad \text { with } \quad \hat{A}_{0}(\hat{\boldsymbol{V}})^{-1}\left[\begin{array}{ccc}
\frac{1}{H^{\prime}} & 0 & -\frac{u}{H^{\prime}} \\
0 & \frac{1}{H^{\prime}} & -\frac{v}{H^{\prime}} \\
0 & 0 & \frac{\sqrt{g H^{\prime}}}{H^{\prime}}
\end{array}\right]
$$

Matrix $\hat{A}_{0}$ and their inverse are well defined as $H^{\prime}$ is always strictly positive.

Multiplying to the left all terms defined in $(3.12)$ by $\hat{A}_{0}^{-1}(\hat{\boldsymbol{V}})$, we finally obtain the symmetric system:

$$
\frac{\partial \boldsymbol{U}}{\partial t}+S_{1}(\boldsymbol{U}) \frac{\partial \boldsymbol{U}}{\partial x}+S_{2}(\boldsymbol{U}) \frac{\partial \boldsymbol{U}}{\partial y}=F(t, x, y, \boldsymbol{U}),
$$

where:

and

$$
S_{1}(\boldsymbol{U})=\left[\begin{array}{ccc}
u & 0 & \frac{H_{g}}{2} \\
0 & u & 0 \\
\frac{H_{g}}{2} & 0 & u
\end{array}\right], \quad S_{2}(\boldsymbol{U})=\left[\begin{array}{ccc}
v & 0 & 0 \\
0 & v & \frac{H_{g}}{2} \\
0 & \frac{H_{g}}{2} & v
\end{array}\right]
$$

$$
F(t, x, y, \boldsymbol{U})=\left(\begin{array}{c}
l v-\frac{4 g \boldsymbol{s}_{\mathbf{s u p} \mid x}}{H_{g}^{2}}-\frac{4 g k u}{H_{g}^{2}\left(1+\frac{k H_{g}^{2}}{12 g \mu}\right)} \\
-l u-\frac{4 g \boldsymbol{s}_{\mathbf{s u p}_{\mid y}}}{H_{g}^{2}}-\frac{4 k v}{H_{g}^{2}\left(1+\frac{k H_{g}^{2}}{12 g \mu}\right)} \\
0
\end{array}\right) .
$$

We will refer to the symmetric system (3.14) as two-dimensional Symmetric Shallow Water system, briefly denoted by $S S W 2 d$ in the sequel. By components, in nonconservative form on $(0, T] \times \Omega$ it reads:

$$
\left\{\begin{array}{l}
\frac{\partial u}{\partial t}+u \frac{\partial u}{\partial x}+v \frac{\partial u}{\partial y}+\frac{H_{g}}{2} \frac{\partial H_{g}}{\partial x}-l v+\frac{4 g \boldsymbol{s}_{\mathbf{s u p} \mid x}}{H_{g}^{2}}+\frac{4 g k u}{H_{g}^{2}\left(1+\frac{k H_{g}^{2}}{12 g \mu}\right)}=0 \\
\frac{\partial v}{\partial t}+u \frac{\partial v}{\partial x}+v \frac{\partial v}{\partial y}+\frac{H_{g}}{2} \frac{\partial H_{g}}{\partial y}+l u+\frac{4 g \boldsymbol{s}_{\mathbf{s u p}} \mid y}{H_{g}^{2}}+\frac{4 g v}{H_{g}^{2}\left(1+\frac{k H_{g}^{2}}{12 g \mu}\right)}=0 \\
\frac{\partial H_{g}}{\partial t}+\nabla \cdot\left(\boldsymbol{v} H_{g}\right)-\frac{H_{g}}{2} \nabla \cdot \boldsymbol{v}=0
\end{array}\right.
$$

where $\boldsymbol{v}=(u, v)^{T}$.

In the following, we will always deal with the SSW2d system together with initial and boundary conditions $(2.28),(2.29)$. Let us now show some analytical properties. Of course, as soon as $\nabla p_{a}=O(\varepsilon)$, the solutions of (3.17) may be interpreted as the solutions of the previously SW2d model.

Proposition 3.2. The eigenvalues of system SSW2d are given by:

$$
\lambda_{2}(\boldsymbol{\xi}, \boldsymbol{U})=\xi_{1} u+\xi_{2} v, \quad \lambda_{1,3}(\boldsymbol{\xi}, \boldsymbol{U})=\xi_{1} u+\xi_{2} v \mp \frac{H_{g}}{2} \sqrt{\xi_{1}^{2}+\xi_{2}^{2}}
$$

and the SSW2d system is a quasilinear strictly hyperbolic system. 
In fact, as $H_{g}>0$ (see (3.11)), we have that

$$
\lambda_{1}(\boldsymbol{\xi}, \boldsymbol{U})<\lambda_{2}(\boldsymbol{\xi}, \boldsymbol{U})<\lambda_{3} .(\boldsymbol{\xi}, \boldsymbol{U}) .
$$

Then the model is (quasilinear) hyperbolic. It is also important to note that the eigenvalues of the SSW2d system are the same as the eigenvalues of the SW2d system.

Remark 3.2 (boundary conditions). If $0<u<H_{g} / 2$, then $\boldsymbol{\Gamma}_{\text {in }}$ and $\boldsymbol{\Gamma}_{\text {out }}$ defined in (2.9) are non-characteristic boundaries for SSW2d model. Further, on $\boldsymbol{\Gamma}_{\mathbf{i n}}$ the number of positive eigenvalues is $p=2$, while on $\boldsymbol{\Gamma}_{\text {out }}$ we have $p=1$. For all $\boldsymbol{x}_{\mathbf{0}} \in \boldsymbol{\Gamma}_{\mathbf{i n}}^{\circ}$, take $\boldsymbol{\xi}=\boldsymbol{n}_{\mathbf{i n}}\left(\boldsymbol{x}_{\mathbf{0}}\right)=(1,0)$; from (3.18) we have:

$$
\lambda_{1}(\boldsymbol{\xi}, \boldsymbol{U})=u-\frac{H_{g}}{2}, \quad \lambda_{2}(\boldsymbol{\xi}, \boldsymbol{U})=u, \quad \lambda_{3}(\boldsymbol{\xi}, \boldsymbol{U})=u+\frac{H_{g}}{2},
$$

while for all $\boldsymbol{x}_{\mathbf{0}} \in \boldsymbol{\Gamma}_{\text {out }}^{\circ}$, take $\boldsymbol{\xi}=\boldsymbol{n}_{\text {out }}\left(\boldsymbol{x}_{\mathbf{0}}\right)=(-1,0)$ :

$$
\lambda_{1}(\boldsymbol{\xi}, \boldsymbol{U})=-u-\frac{H_{g}}{2}, \quad \lambda_{2}(\boldsymbol{\xi}, \boldsymbol{U})=-u, \quad \lambda_{3}(\boldsymbol{\xi}, \boldsymbol{U})=-u+\frac{H_{g}}{2} .
$$

On the other side, from boundary conditions (2.28), we have that $\boldsymbol{\Gamma}_{\boldsymbol{c}}$ defined in (2.10) is a characteristic boundary for SSW2d model on our bounded domain (2.2). Further on, we have on $\boldsymbol{\Gamma}_{\boldsymbol{c}}$ exactly a null, a positive and a negative eigenvalue. For all $\boldsymbol{x}_{\mathbf{0}} \in \stackrel{\circ}{\boldsymbol{\Gamma}}_{\boldsymbol{c}}$, take $\boldsymbol{\xi}=(0, \pm 1)$; from (3.18) we have:

$$
\lambda_{1}(\boldsymbol{\xi}, \boldsymbol{U})= \pm v-\frac{H_{g}}{2} \quad \lambda_{2}(\boldsymbol{\xi}, \boldsymbol{U})= \pm v, \quad \lambda_{3}(\boldsymbol{\xi}, \boldsymbol{U})= \pm v+\frac{H_{g}}{2} .
$$

In the following, we will always consider for the sake of simplicity the following so-called subsonic ${ }^{1}$ hypothesis:

$$
u \in\left(0, \frac{H_{g}}{2}\right), \quad|v| \in\left[0, \frac{H_{g}}{2}\right) .
$$

\subsection{Viscous perturbation of the SSW2d model}

Let us consider a viscous perturbations of the SSW2d system (3.14), defined on the bounded domain $\Omega(2.2)$ in the evolution time interval $[0, T]$. To each equation of the SSW2d system we add a Laplacian term multiplied by the artificial viscosity coefficient $\nu$ and we complete the system with initial conditions and the following Dirichlet boundary conditions:

$$
\left\{\begin{array}{l}
\frac{\partial \boldsymbol{U}^{\nu}}{\partial t}+S_{1}\left(\boldsymbol{U}^{\nu}\right) \frac{\partial \boldsymbol{U}^{\nu}}{\partial x}+S_{2}\left(\boldsymbol{U}^{\nu}\right) \frac{\partial \boldsymbol{U}^{\nu}}{\partial y}-\nu \Delta \boldsymbol{U}^{\nu}=F\left(t, x, y, \boldsymbol{U}^{\nu}\right) \quad \text { on } \Omega \times(0, T], \\
\boldsymbol{U}_{\Gamma}^{\nu}=\boldsymbol{a} \text { on } \boldsymbol{\Gamma} \times[0, T] \\
\boldsymbol{U}_{\mid t=0}^{\nu}=\boldsymbol{U}_{0} \quad \text { on } \Omega \times\{t=0\}
\end{array}\right.
$$

where $\boldsymbol{a}(t, x, y)$ is a function to be prescribed on $\boldsymbol{\Gamma} \times(0, T]$ and taking its values in $\mathbb{R}^{3}$. We used here as in (2.14) the same function-name $\boldsymbol{a}$, to indicate Dirichlet conditions for a viscous problem (system NS3d (2.3) or the above viscous perturbed SSW2d system (3.21)); of course, we are dealing with two different functions, even if they show some logical relationship.

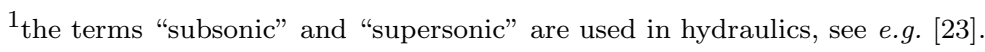


At present, an existence and uniqueness result global in time for smooth solutions of parabolic problems like (3.21) is available (see e.g. Ladyzenskaia, Solonnikov, Ural'ceva [17], Th. 7.1, Chap. VII, pp. 596-597) only for a smooth and bounded boundary, with initial and boundary conditions sufficiently smooth, satisfying suitable compatibility conditions.

However, as far as we know, existence and uniqueness results for initial boundary value hyperbolic problems like:

$$
\left\{\begin{array}{l}
\frac{\partial \boldsymbol{U}}{\partial t}+S_{1}(\boldsymbol{U}) \frac{\partial \boldsymbol{U}}{\partial x}+S_{2}(\boldsymbol{U}) \frac{\partial \boldsymbol{U}}{\partial y}=F(t, x, y, \boldsymbol{U}) \quad \text { on } \Omega \times(0, T] \\
\boldsymbol{U}_{\Gamma} \in \mathcal{C}(\boldsymbol{a}) \quad \text { on } \boldsymbol{\Gamma} \times(0, T] \\
\boldsymbol{U}_{\mid t=0}=\boldsymbol{U}_{0} \quad \text { on } \Omega \times\{t=0\}
\end{array}\right.
$$

and results on convergence of (3.21) to (3.22) in a suitable space-time norm, are not yet available on a bounded domain like (2.2), even if one smooths its edges.

When the domain is a half-space in $\mathbb{R}^{n}$, some literature is available on the convergence as $\nu \rightarrow 0$ of perturbed symmetric problems like (3.21) to hyperbolic IVB problems like (3.22).

Let us observe that we may consider our bounded domain $\Omega(2.2)$ as the intersection of four half-spaces:

$$
\Omega=\Omega_{\text {in }} \cap \Omega_{\text {out }} \cap \Omega_{c+} \cap \Omega_{c-},
$$

where, recalling Figure 2,

$$
\begin{aligned}
& \Omega_{\text {in }}=\left\{(x, y) \in \mathbb{R}^{2} \quad \text { s.t. } \quad x>x_{\text {in }}\right\}, \quad \Omega_{\text {out }}=\left\{(x, y) \in \mathbb{R}^{2} \quad \text { s.t. } \quad x<x_{\text {out }}\right\}, \\
& \Omega_{c+}=\left\{(x, y) \in \mathbb{R}^{2} \quad \text { s.t. } y>-L / 2\right\}, \quad \Omega_{c-}=\left\{(x, y) \in \mathbb{R}^{2} \quad \text { s.t. } y<L / 2\right\} .
\end{aligned}
$$

If conditions (3.20) are satisfied and, in view of boundary conditions (2.28), $\Omega_{\text {in }}$ and $\Omega_{\text {out }}$ have non-characteristic boundaries, while $\Omega_{c+}$ and $\Omega_{c-}$ may have characteristic boundaries. We will consider separately first the noncharacteristic case, then the characteristic case (if $v=0$ ).

(1) The non-characteristic case: if we set our second order viscous perturbed SSW2d model (3.21) into a half-space of the form $\Omega_{\text {in }}$ (or $\Omega_{\text {out }}$ ), then by [17], we have that (3.21) has a unique smooth solution $\boldsymbol{U}^{\nu}$ globally in time, while by [20] the hyperbolic problem (3.22) has a unique smooth solution $\boldsymbol{U}^{0}$ locally in time. Thanks to Grenier and Guès in [10], we may also conclude that there exists a time $T_{0}$ such that the following convergence result is valid:

$$
\left\|\boldsymbol{U}^{\nu}-\boldsymbol{U}\right\|_{L^{2}\left(\left(0, T_{0}\right) \times \Omega\right.} \rightarrow 0 \quad \text { as } \nu \rightarrow 0 .
$$

A deeper analysis of the compatibility between the theory exposed by Grenier and Guès in [10] and our case is shown in [5].

(2) The characteristic case: for this case, literature is not so extensive as in the previous one. We have a result by Guès in [11] for the semi linear case. In this case, we don't have much information on the admissible set of boundary conditions for the hyperbolic inviscid limit, as we had in the noncharacteristic case. Another result, by Grenier in [9], deals with the quasilinear case, but only in the case of a scalar problem.

Let us end this section by remarking that the classical Shallow Water model (2.41) is an hyperbolic model as well, and that it may be symmetrized the same way as our SW2d model; we shall refer to this one as to the CSSW2d model. In terms of variable $\boldsymbol{U}^{*}=\left(u, v, H_{g}^{*}\right)^{T}$, where we posed:

$$
H_{g}^{*}=2 \sqrt{g H}
$$

The CSSW2d model on $(0, T) \times \Omega$ reads:

$$
\frac{\partial \boldsymbol{U}^{*}}{\partial t}+S_{1}\left(\boldsymbol{U}^{*}\right) \frac{\partial \boldsymbol{U}^{*}}{\partial x}+S_{2}\left(\boldsymbol{U}^{*}\right) \frac{\partial \boldsymbol{U}^{*}}{\partial y}=G\left(t, x, y, \boldsymbol{U}^{*}\right)
$$


where matrices $S_{1}$ and $S_{2}$ are the same as in (3.15), while the right hand side $G$ is now given by:

$$
G\left(t, x, y, \boldsymbol{U}^{*}\right)=\left(\begin{array}{c}
l v-\frac{\partial p_{a}}{\partial x}+\frac{4 g s_{\mathbf{s u p} \mid x}}{H^{* 2}}-\frac{4 g^{2} k u|\boldsymbol{v}|}{H^{* 2}} \\
-l u-\frac{\partial p_{a}}{\partial y}+\frac{4 g s_{\mathbf{s u p} \mid y}}{H_{g}^{* 2}}-\frac{4 g^{2} k v|\boldsymbol{v}|}{H_{g}^{* 2}} \\
0
\end{array}\right)
$$

assuming that $H_{g}^{*}>0$ iff $H>0$.

Remark 3.3. About the introduction of a viscous perturbation, we want to mention the paper by Sammartino and Caflisch in [21]. They consider the convergence of the three-dimensional incompressible Navier-Stokes equations to the inviscid incompressible Euler equations; the physical second order viscous term which appears in the Navier-Stokes equation is considered to be vanishing, thus "perturbing" the momentum equation in the Euler system, but the continuity equation is left unaltered. In our paper, instead, following Grenier and Guès in [10], we consider an artificial second order viscous perturbation of all the equations of a hyperbolic system (with suitable properties).

\section{NumericAl EXPERIMENTS}

In this section we present an academic test case in order to compare the results of the proposed model with the results obtained with the viscous perturbed CSSW2d model, when the wind and the Coriolis effects are neglected $\left(s_{\sup \mid x}=s_{\sup _{\mid y}}=0\right.$ and $\left.l=0\right)$. Numerical approximation is based on the space-time approximation introduced in [6]: in particular, we consider piecewise-quadratic finite element in space and a first order semiimplicit finite difference scheme in time. Notice that on the test cases, we will refer to the MKS system of measurement; for the sake of simplicity, we will often omit in the following the unit of measurement.

All the numerical results are obtained using the $\mathrm{C}++$ library FreeFem $++[13]$.

\subsection{The Gaussian-hill test case}

We deal with the spreading of a body of water initially having a Gaussian-hill distribution. The closed water basin is $\Omega=[0,10] \times[0,10]$ with a flat bathymetry $b(x, y)=-1$. The final time is $T=0.81$. Initial conditions are:

$$
C(x, y, 0)=5+5 \exp \left(\left(-(x-5)^{2}-(y-5)^{2}+1\right)\right), u_{0}=0, v_{0}=0,
$$

while for any time $t \in(0, T]$ the boundary conditions are given by:

$$
u=0, v=0, C=5 .
$$

The variable $C$ is $H_{g}$ (see (3.10)) when the SSW2d model is considered, $H_{g}^{*}$ (see (3.24)) for the CSSW2d model. Remark that in the viscous perturbed SSW2d model, the atmospheric pressure term is comprised into the celerity term $H_{g}$, while in the viscous perturbed CSSW2d model, pressure is considered apart from the celerity variable $H_{g}^{*}$.

On a uniform triangular grid (with space-step equal to 0.5) we compare the different behaviors of our perturbed SSW2d model with the viscous perturbed CSSW2d model, considering different values of the artificial 
viscosity parameter $\nu$; we first point out the difference in treating the friction term (letting the atmospheric pressure be a constant) and the atmospheric-pressure term, considering an atmospheric pressure wave acting on the Gaussian-hill. In both cases we neglect for simplicity the wind effect and the Coriolis force effect; we set the time-step $\Delta t=0.001$, the final evolution time $T=0.81$ and the physical viscosity parameter $\mu=0.2$.

In the following simulations we will compare the isovalues of the elevation $\eta$ (see the beginning of Section 2) at the final time $t=T$ with the vertical cuts of the elevation profiles in $y=5$ at $t=T$ obtained by the viscous perturbed SSW2d model and the viscous perturbed CSSW2d model with artificial viscosity values $\nu=0.1$ and $\nu=0.00001$ respectively.

Friction effects. Let us choose $k=50$ and $p_{a}=1$. The initial plotting of elevation in the case of our model is very similar to that for the classical model, as the atmospheric pressure is supposed to be a constant.

Remark that our model evolves more rapidly than the classical one; in fact at the final time (Fig. 4a, Fig. 4c and Fig. 5a, Fig. 5c) we notice that the wave depicted by our model is already propagating toward the boundary, loosing much of its initial elevation, while the wave depicted by the classical model (Fig. 4b, Fig. 4d and Fig. 5b, Fig. 5d) is still going down from its initial elevation. It is clear that the classical model is quasi-insensitive with respect to variations of the artificial viscosity, while our model is very sensitive to variations on the artificial viscosity. Physically, water, which is an almost inviscid flow, behaves near to simulations with an artificial viscosity $\nu=0.00001$, which is a value of the same magnitude order of water physical viscosity. This fact is in accordance with respect to the convergence theory recalled in Section 3.1 (even if in particular cases) and with respect to our accurate model derivation exposed in Section 1.

Atmospheric pressure effects. In order to point out atmospheric pressure effects, let us consider an experiment where $k=0$ and atmospheric pressure is given by:

$$
p_{a}=x+t
$$

Since atmospheric pressure is not constant, the initial plotting of elevation for the viscous perturbed CSSW2d model is just the same as if pressure is constant (as the classical model is pressure independent). But in the case of our SSW2d model, where elevation takes the atmospheric pressure into account, then the plotting of initial elevation is quite different from the constant-pressure case (compare Fig. 6a with Fig. 6b). We observe that elevation $\eta$ might take negative values, as it is in the case of our model (see Fig. 7a, Fig. 7c and Fig. 8a, Fig. 8c). In this situation, both the perturbed CSSW2d (see Fig. 7b, Fig. 7d and Fig. 8b, Fig. 8d) and the perturbed SSW2d model are sensitive to the change of artificial viscosity. In particular for $\nu=0.00001$ both the perturbed SSW2d model and the perturbed CSSW2d model behave much less regularly as for $\nu=0.1$. Notice that for a low value of the artificial viscosity parameter $\nu$, some peaks are generated. Observing the wave profiles, one may conclude that results obtained by our model look closer to physical expectation than the classical one. As a pressure wave moving horizontally toward the positive orientation of $x$-axis is acting, one expects the wave to be "lower" on that side; that is what happens by our new model, while the classical model behaves thoroughly unphysically.

\section{Conclusions And OPEn PROBlems}

We newly derived a two-dimensional Shallow Water model (SW2d model) on a bounded domain and set physical boundary conditions on velocity. A specification of the "nature" (characteristic, non-characteristic) of boundary edges is set.

An open problem is to demonstrate the convergence of the artificial viscous-perturbed model to our model on a two-dimensional domain, with inhomogeneous initial and boundary data and with different combinations of characteristic and non-characteristic edges. It would be also interesting to find out what is the time-interval while our system evolves smoothly, in relation with initial and boundary data and supposing that we are in the subsonic assumptions (3.20). Another task which could be performed, is to find out some relations between 


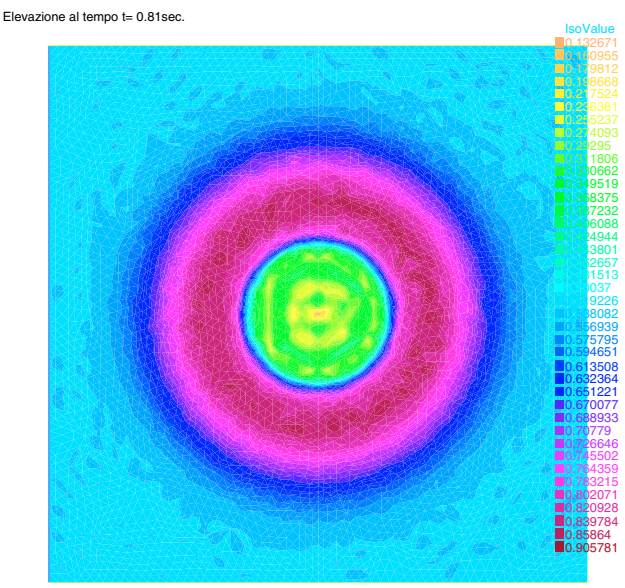

(a)

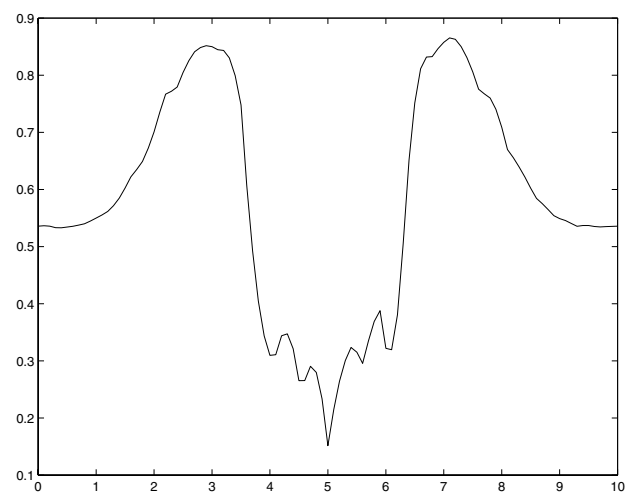

(c)

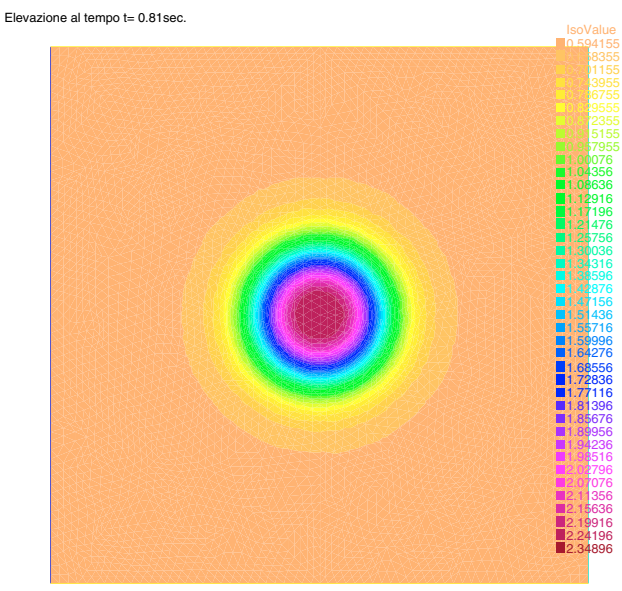

(b)

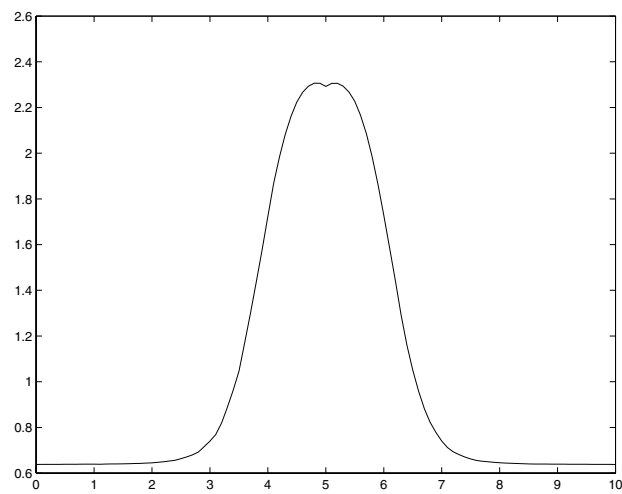

(d)

Figure 4. (a) Final total elevation for the Gaussian-hill case: the viscous perturbed SSW2d model, $\nu=0.00001$, friction $k=50$, atmospheric pressure $p_{a}=1$. (b) Final total elevation for the Gaussian-hill case: the viscous perturbed CSSW2d model, $\nu=0.00001$, friction $k=50$, atmospheric pressure $p_{a}=1$. (c) Elevation profile of the final total elevation for the Gaussianhill case: the viscous perturbed SSW2d model, $\nu=0.00001$, friction $k=50$, atmospheric pressure $p_{a}=1$. (d) Elevation profile of the final total elevation for the Gaussian-hill case: the viscous perturbed CSSW2d model, $\nu=0.00001$, friction $k=50$, atmospheric pressure $p_{a}=1$.

initial and boundary conditions such that the subsonic hypothesis, which are supposed a priori, are effectively point-wise fulfilled.

Acknowledgements. We thank warmly Dr. Debora Amadori (Università Statale degli Studi dell'Aquila, Dipartimento di Matematica Pura e Applicata) for the substantial help she gave us in structuring the paper and in punctualizing the analytical setting. We are also grateful to Prof. Marco Sammartino (Università Statale degli Studi di Palermo, Dipartimento di Matematica e Applicazioni): his contribution in verifying our model has been of greatest help. We finally owe much gratitude to Prof. Benoît Perthame (École Normale Supérieure - DMA, INRIA Rocquencourt) for the many suggestions he gave us in order to improve the present work. 


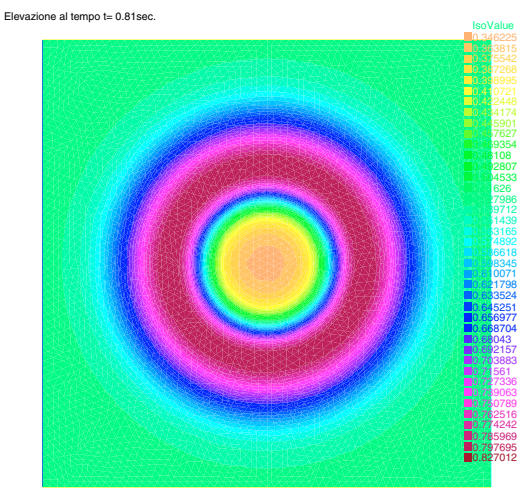

(a)

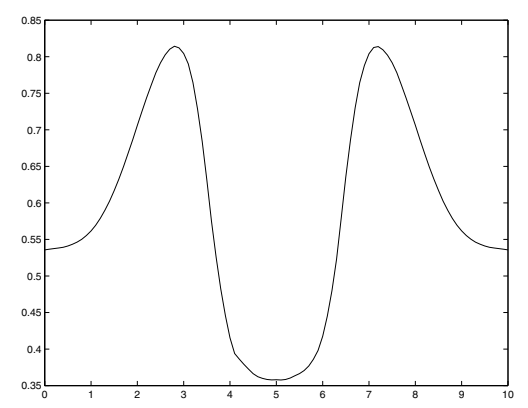

(c)

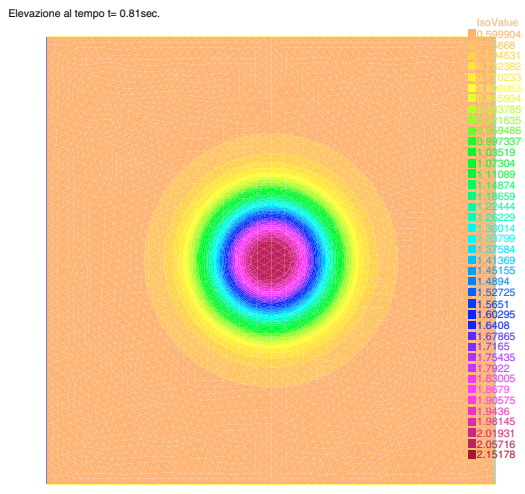

(b)

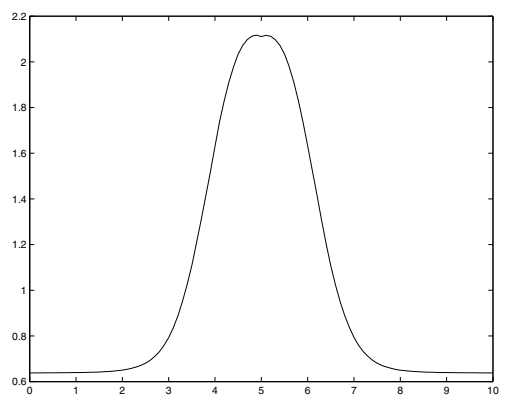

(d)

Figure 5. (a) Final total elevation for the Gaussian-hill case: the viscous perturbed SSW2d model, $\nu=0.1$, friction $k=50$, atmospheric pressure $p_{a}=1$. (b) Final total elevation for the Gaussian-hill case: the viscous perturbed CSSW2d model, $\nu=0.1$, friction $k=50$, atmospheric pressure $p_{a}=1$. (c) Elevation profile of the final total elevation for the Gaussian-hill case: the viscous perturbed SSW2d model, $\nu=0.1$, friction $k=50$, atmospheric pressure $p_{a}=1$. (d) Elevation profile of the final total elevation for the Gaussian-hill case: the viscous perturbed CSSW2d model, $\nu=0.1$, friction $k=50$, atmospheric pressure $p_{a}=1$.

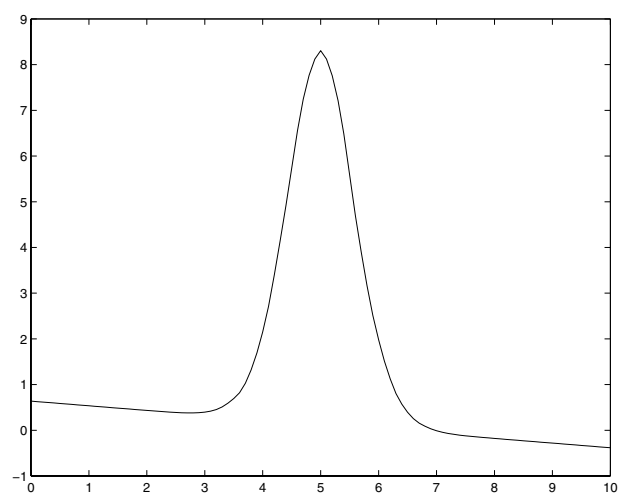

(a)

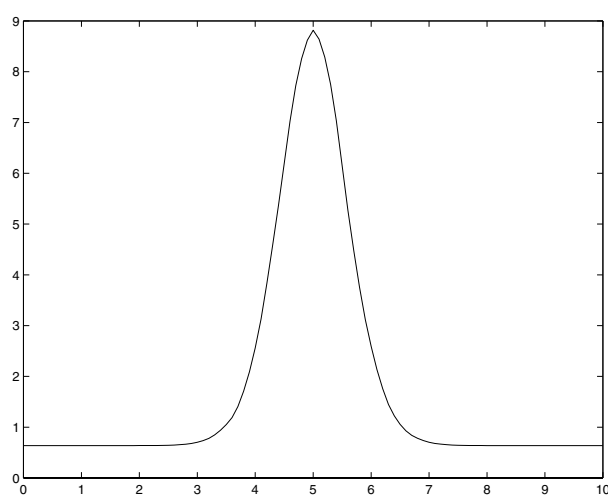

(b)

FIGURE 6. (a) Initial total elevation for the Gaussian-hill case: the viscous perturbed SSW2d model, atmospheric pressure $p_{a}=x+t$. (b) Initial total elevation profile for the Gaussian-hill case: the viscous perturbed CSSW2d model, atmospheric pressure $p_{a}=x+t$. 


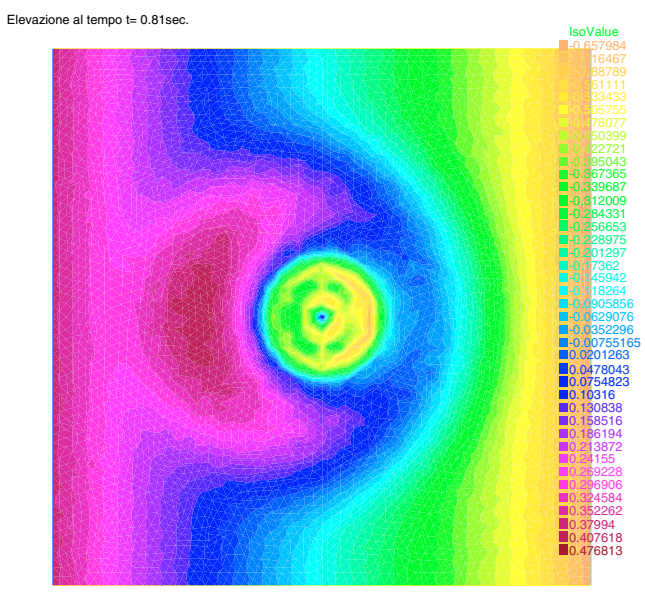

(a)

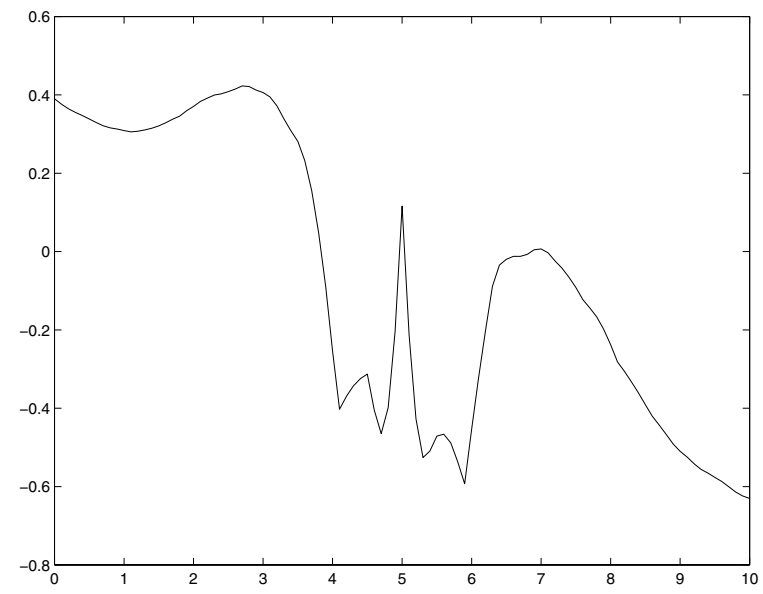

(c)

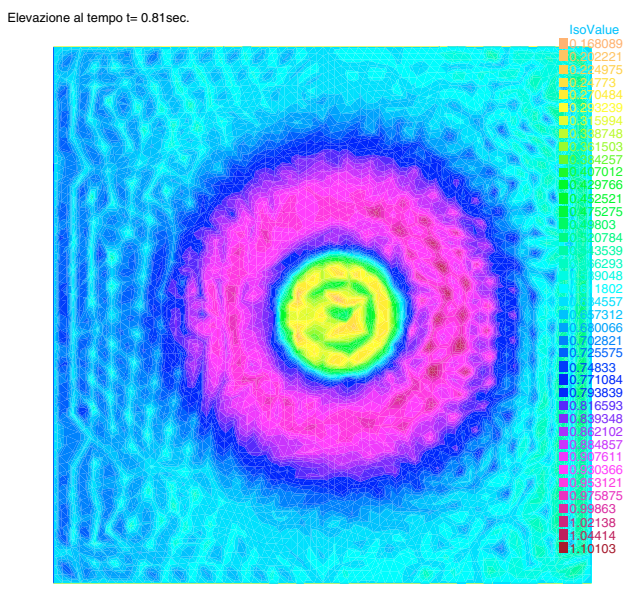

(b)

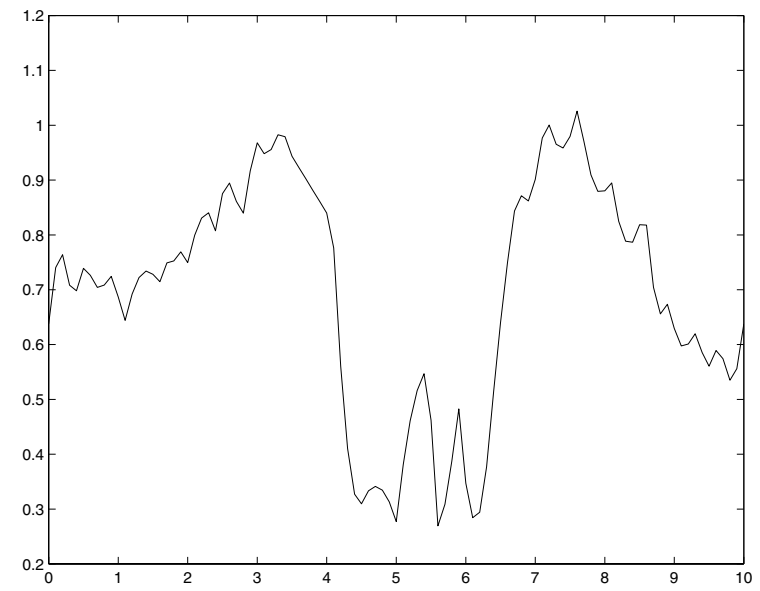

(d)

FIGURE 7. (a) Final total elevation for the Gaussian-hill case: the viscous perturbed SSW2d model, $\nu=0.00001$, friction $k=0$, atmospheric pressure $p_{a}=x+t$. (b) Final total elevation for the Gaussian-hill case: the viscous perturbed CSSW2d model, $\nu=0.00001$, friction $k=0$, atmospheric pressure $p_{a}=x+t$. (c) Elevation profile of the final total elevation for the Gaussianhill case: the viscous perturbed SSW2d model, $\nu=0.00001$, friction $k=0$, atmospheric pressure $p_{a}=x+t$. (d) Elevation profile of the final total elevation for the Gaussian-hill case: the viscous perturbed CSSW2d model, $\nu=0.00001$, friction $k=0$, atmospheric pressure $p_{a}=x+t$. 


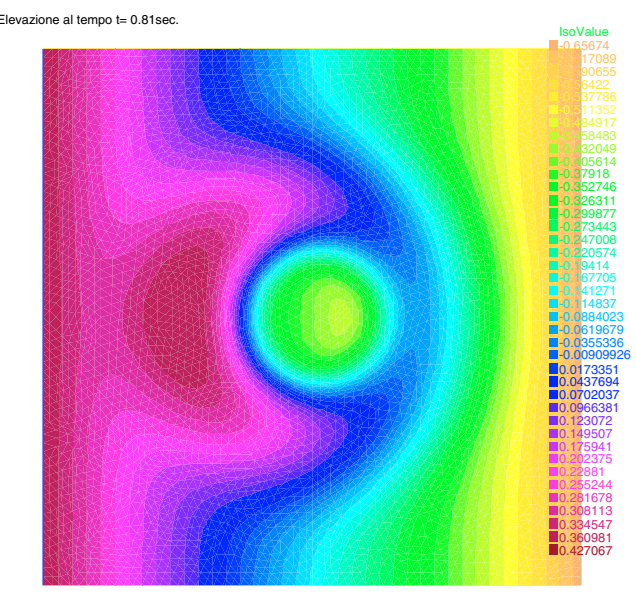

(a)

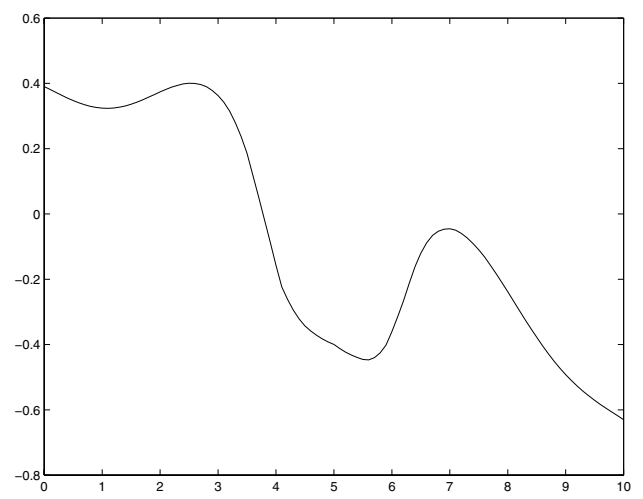

(c)

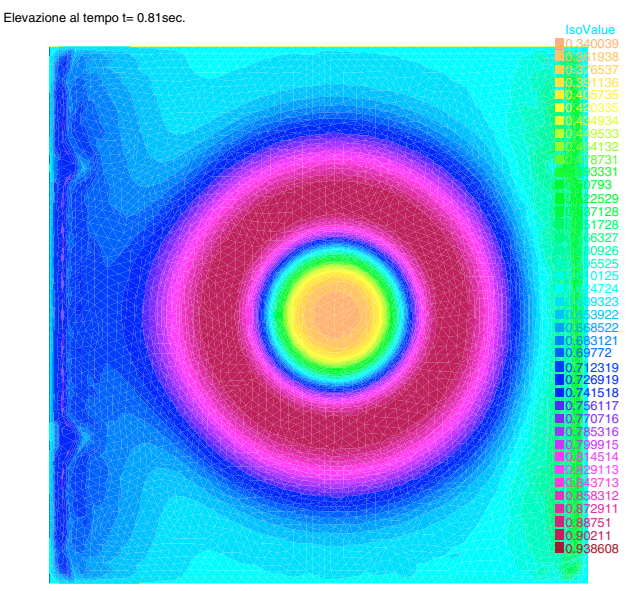

(b)

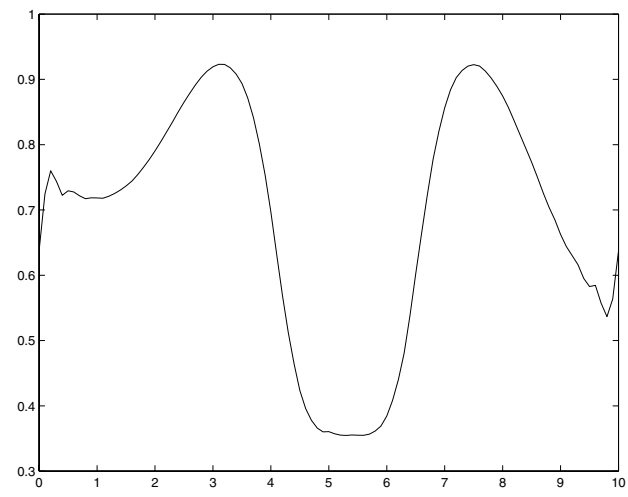

(d)

Figure 8. (a) Final total elevation for the Gaussian-hill case: the viscous perturbed SSW2d model, $\nu=0.1$, friction $k=0$, atmospheric pressure $p_{a}=x+t$. (b) Final total elevation for the Gaussian-hill case: the viscous perturbed CSSW2d model, $\nu=0.1$, friction $k=0$, atmospheric pressure $p_{a}=x+t$. (c) Elevation profile of the final total elevation for the Gaussian-hill case: the viscous perturbed SSW2d model, $\nu=0.1$, friction $k=0$, atmospheric pressure $p_{a}=x+t$. (d) Elevation profile of the final total elevation for the Gaussian-hill case: the viscous perturbed CSSW2d model, $\nu=0.1$, friction $k=0$, atmospheric pressure $p_{a}=x+t$. 


\section{REFERENCES}

[1] V.I. Agoshkov, D. Ambrosi, V. Pennati, A. Quarteroni and F. Saleri, Mathematical and numerical modelling of shallow water flow. Comput. Mech. 11 (1993) 280-299.

[2] V.I. Agoshkov, A. Quarteroni and F. Saleri, Recent developments in the numerical simulation of shallow water equations. Boundary conditions. Appl. Numer. Math. 15 (1994) 175-200.

[3] J.P. Benque, J.A. Cunge, J. Feuillet, A. Hauguel and F.M. Holly, New method for tidal current computation. J. Waterway, Port, Coastal and Ocean Division, ASCE 108 (1982) 396-417.

[4] J.P. Benque, A. Haugel and P.L. Viollet, Numerical methods in environmental fluid mechanics. M.B. Abbot and J.A. Cunge Eds., Eng. Appl. Comput. Hydraulics II (1982) 1-10.

[5] S. Ferrari, A new two-dimensional Shallow Water model: physical, mathematical and numerical aspects Ph.D. Thesis, a.a. 2002/2003, Dottorato M.A.C.R.O., Università degli Studi di Milano.

[6] S. Ferrari, Convergence analysis of a space-time approximation to a two-dimensional system of Shallow Water equations. Internat. J. Appl. Analysis (to appear).

[7] J.F. Gerbeau and B. Perthame, Derivation of viscous Saint-Venant system for laminar shallow water; numerical validation. Discrete Contin. Dyn. Syst. Ser. B 1 (2001) 89-102.

[8] R.H. Goodman, A.J. Majda and D.W. Mclaughlin, Modulations in the leading edges of midlatitude storm tracks. SIAM J. Appl. Math. 62 (2002) 746-776.

[9] E. Grenier, Boundary layers for parabolic regularizations of totally characteristic quasilinear parabolic equations. J. Math. Pures Appl. 76 (1997) 965-990.

[10] E. Grenier and O. Guès, Boundary layers for viscous perturbations of noncharacteristic quasilinear hyperbolic problems. $J$. Differential Equations 143 (1998) 110-146.

[11] O. Guès, Perturbations visqueuses de problèmes mixtes hyperboliques et couches limites. Grenoble Ann. Inst. Fourier 45 (1995) 973-1006.

[12] M.E. Gurtin, An introduction to continuum mechanics. Academic Press, New York (1981).

[13] F. Hecht and O. Pironneau, FreeFem++:Manual version 1.23, 13-05-2002. FreeFem++ is a free software available at: http://www-rocq.inria.fr/Frederic.Hecht/freefem++.htm

[14] J.M. Hervouet and A. Watrin, Code TELEMAC (système ULYSSE) : Résolution et mise en œuvre des équations de SaintVenant bidimensionnelles, Théorie et mise en œuvre informatique, Rapport EDF HE43/87.37 (1987).

[15] S. Jin, A steady-state capturing method for hyperbolic systems with geometrical source terms. ESAIM: M2AN 35 (2001) 631-645.

[16] A. Kurganov and L. Doron, Central-upwind schemes for the Saint-Venant system. ESAIM: M2AN 36 (2002) $397-425$.

[17] O.A. Ladyzenskaja, V.A. Solonnikov and N.N. Ural'ceva, Linear and quasilinear equations of parabolic type. Providence, Rhode Island. Amer. Math. Soc. (1968).

[18] D. Levermore and M. Sammartino, A shallow water model with eddy viscosity for basins with varying bottom topography. Nonlinearity 14 (2001) 1493-1515.

[19] E. Miglio, A. Quarteroni and F. Saleri, Finite element approximation of a quasi-3D shallow water equation. Comput. Methods Appl. Mech. Engrg. 174 (1999) 355-369.

[20] J. Rauch and F. Massey, Differentiability of solutions to hyperbolic initial-boundary value problems. Trans. Amer. Math. Soc. 189 (1974) 303-318.

[21] M. Sammartino and R.E. Caflisch, Zero viscosity limit for analytic solutions of the Navier-Stokes equations on a half-space. I. Existence for Euler and Prandtl Equations; II. Construction of the Navier-Stokes solution. Comm. Math. Physics 192 (1998) 433-461 and 463-491.

[22] D. Serre, Sytems of conservation laws. I and II, Cambridge University Press, Cambridge (1996).

[23] G.B. Whitham, Linear and nonlinear waves. John Wiley \& Sons, New York (1974). 